
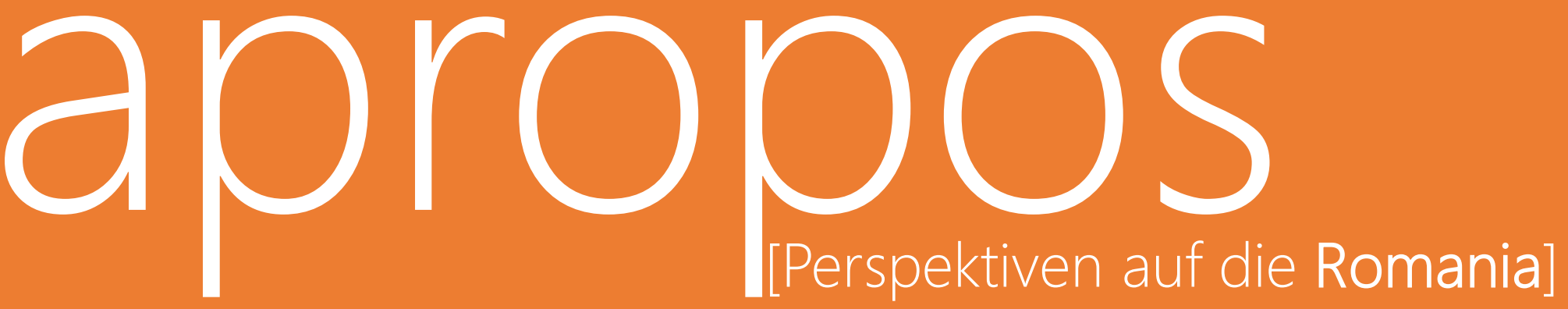

Sprache/Literatur/Kultur/Geschichte/Ideen/Politik/Gesellschaft

Astheure und maintenant im Französischen Nordamerikas im Vergleich.

Eine korpusbasierte Untersuchung

Hans Baumann

apropos [Perspektiven auf die Romania] hosted by Hamburg University Press 2021, 6 pp. 156-183

ISSN: 2627-3446

Online https://journals.sub.uni-hamburg.de/apropos/article/view/1682

Zitierweise

Baumann, Hans. 2021. „Astheure und maintenant im Französischen Nordamerikas im Vergleich. Eine korpusbasierte Untersuchung.", apropos [Perspektiven auf die Romania] 6/2021, 156-183. doi: 10.15460/apropos.6.1682 


\title{
Astheure und maintenant im Französischen Nordamerikas im Vergleich
}

\author{
Eine korpusbasierte Untersuchung
}

\author{
Hans Baumann \\ studiert seit 2017 Französisch und \\ Geschichte auf Lehramt und ist \\ wissenschaftliche Hilfskraft an der \\ Eberhard Karls Universität Tübingen. \\ hans.baumann@student.uni-tuebingen.de
}

\begin{abstract}
Keywords
astheure - maintenant - Vergleich - Nordamerika - Korpuslinguistik - Premiers Travaux

Dieser Artikel basiert auf einer Hausarbeit, die im Rahmen des Seminars „Korpuslinguistik Französisch“ (SoSe 2019, Eberhard Karls Universität Tübingen) entstanden ist. Die Arbeit wurde für die Publikation in der apropos-Rubrik Premiers Travaux unter Betreuung von Apl. Prof. Dr. Rembert Eufe (Akad. Rat für französische, italienische und spanische Sprachwissenschaft am Romanischen Seminar der Eberhard Karls Universität Tübingen) überarbeitet.
\end{abstract}

\section{Einleitung}

1) Ça prend tout mon petit change!

2) C'est-tu loin, ça?

Für einen aus Frankreich stammenden französischsprachigen Sprecher sind diese beiden Sätze nur schwer zu verstehen. Ein aus Nordamerika stammender französischsprachiger Sprecher hingegen hätte kein Problem, diese Sätze zu begreifen. Die Sätze sind nämlich nur zwei von zahlreichen Beispielen, die illustrieren, wie sich das in Nordamerika gesprochene Französisch vom Französischen in Frankreich unterscheidet. Neben phonetisch-phonologischen, morphologischen oder auch syntaktischen Merkmalen unterscheidet sich das Französische Nordamerikas im Vergleich zum Französischen in Frankreich zum Teil stark hinsichtlich des Wortschatzes. ${ }^{1}$ So werden dort neben Entlehnungen aus anderen Sprachen, vor allem

\footnotetext{
${ }^{1}$ Solche lexikalischen Unterschiede zeigen sich in Beispiel 1. Change 'Kleingeld' ist hier aus dem Englischen entlehnt und besitzt eine übertragene Bedeutung. Petit change meint hier daher énergie oder effort. Beispiel 2 illustriert die morphologischen bzw. syntaktischen Unterschiede zum hexagonalen Französisch. In diesem vor allem für das Französische Québecs typischen Beispiel folgt direkt auf das konjugierte Verb die Interrogativpartikel $t u$, die ausschließlich in Entscheidungsfragen verwendet werden kann, ohne jedoch eine Bedeutung zu tragen. Für eine ausführlichere Darstellung der allgemeinen Merkmale des Französischen in Nordamerika cf. Pöll 2017, 89-108.
} 
dem Englischen, und Neologismen auch Archaismen verwendet. Dabei handelt es sich um Wörter, die in Frankreich nicht mehr oder kaum noch verwendet werden, in Nordamerika jedoch gebräuchlich sind. Ein Beispiel hierfür ist das Temporaladverb astheure 'jetzt', ein Synonym für maintenant, wie es der Petit Robert (NPR) angibt (cf. NPR, s. v. heure). Beide Temporaladverbien werden als deiktisches Element für $T_{0}$, den Zeitpunkt des Sprechens oder Schreibens, gebraucht. In Frankreich wird astheure nur noch regionalsprachlich verwendet, im französischsprachigen Teil Nordamerikas ist es hingegen flächendeckend verbreitet. Im Rahmen dieses Aufsatzes soll korpusbasiert untersucht werden, wie das Wort astheure in Nordamerika im Vergleich zu seinem Synonym maintenant verwendet wird.

Bisher gibt es in der Forschung im Gegensatz zu anderen für das nordamerikanische Französisch typischen Wörtern kaum Arbeiten zu astheure. ${ }^{2} \mathrm{Zu}$ den wenigen Beiträgen zählen beispielsweise jene von Mathieu Avanzi (2019), Benoît Melançon (2016) oder auch von Aude Séguin (2011). Allerdings handelt es sich bei den beiden erstgenannten Arbeiten lediglich um wissenschaftlich fundierte Blogeinträge. Darüber hinaus wird das Wort in zahlreichen Wörterbüchern, Grammatiken und sonstigen Abhandlungen zum Französischen in Nordamerika erwähnt. In vielen wissenschaftlichen Beiträgen bleibt es jedoch zumeist bei der bloßen Nennung, ohne dass näher auf astheure eingegangen wird. Zumeist wird lediglich hervorgehoben, dass es sich um ein Synonym von maintenant handle, welches vor allem im französischsprachigen Teil Nordamerikas verwendet wird.

Weder die Wörterbucheinträge noch die wenigen bislang veröffentlichten Arbeiten zu astheure untersuchen dessen Verwendung anhand von Korpusabfragen. Dabei scheint es durchaus sinnvoll, eine solche Untersuchung auf korpuslinguistische Ergebnisse zu stützen, da die an der Schriftlichkeit orientierte Art der Beschreibung in Wörterbucheinträgen häufig zu einer Vernachlässigung der Mündlichkeit führt, die bei astheure allerdings nicht außer Acht gelassen werden darf. Durch die korpuslinguistischen Ergebnisse kann das Lemma astheure außerdem als Lexem im Kontext analysiert werden und muss nicht losgelöst von seiner Verwendungsweise betrachtet werden, wie dies unter anderem in Wörterbucheinträgen der Fall ist (cf. Schafroth 2014, 81). Die in diesem Aufsatz verwendeten authentischen Korpusbelege können so zur "Natürlichkeit eines Beispiels und seiner Nähe an der Realität des Sprachgebrauchs bei[tragen]" (Herbst/Klotz 2003, 58). ${ }^{3}$ Des Weiteren wurden bislang keine Vergleiche hinsichtlich der Verwendung von astheure und maintenant angestellt. An diesen Punkten möchte der Aufsatz ansetzen. Er nimmt sich zum Ziel,

\footnotetext{
2 Dies zeigt bereits ein Blick auf den Index lexicologique québécois des Trésor de la langue française au Québec. Hier nämlich ergibt die Suche für andere typische Wörter für das nordamerikanische Französisch wie icitte deutlich mehr Belege in wissenschaftlichen Publikationen. Während für astheure 69 Belege zu finden sind, weist icitte 130 Belege auf. Auch typische Substantive wie char (415 Belege) kommen deutlich häufiger vor als astheure.

${ }^{3}$ Anhand von Korpusbelegen wie Ils restont à Montréal astheure encore euh (Korpus FRAN: Conversation entre les sœurs Germaine (68 ans) et Victoire (66 ans), à Moncton; 1976), die Wörterbücher zur Veranschaulichung der Verwendung von astheure so nicht abbilden würden, in denen sich jedoch die „Natürlichkeit eines Beispiels" widerspiegelt, kann auf diese Weise auch das Auftreten von astheure in Kombination mit weiteren sprachlichen Besonderheiten des nordamerikanischen Französisch (hier bspw. die Verbalendung -ont in der 3. Person Plural) erkannt werden (cf. dazu auch Schafroth 2014, 81).
} 
das Wort astheure, das in der Forschung bisher nur marginal berücksichtigt wurde, näher zu untersuchen. Dabei möchte der Aufsatz zu einem doppelten Erkenntnisgewinn gelangen: Zum einen soll die Verwendung von astheure anhand von Korpusabfragen in authentischen Kontexten erfasst und dadurch genauer beschrieben werden, zum anderen sollen diese Ergebnisse mit der Verwendung von maintenant kontrastiert werden.

Zur Untersuchung der genannten Fragestellung soll zunächst ein Blick auf das Wort astheure geworfen werden. Neben seiner Etymologie sollen in einem ersten Schritt auch Wörterbucheinträge zu astheure analysiert werden, um einen ersten Überblick zu erhalten. In einem zweiten Schritt erfolgt die Untersuchung der Verbreitung von astheure sowohl in Frankreich als auch in Nordamerika, um Schlussfolgerungen zur Verbreitung des Wortes in Nordamerika ziehen zu können. Darauf aufbauend sollen dann astheure und maintenant anhand von Korpusabfragen hinsichtlich ihrer Verwendung im nordamerikanischen Französisch miteinander verglichen werden.

Um einen möglichst umfassenden Überblick zur Verwendung der beiden Wörter zu erhalten, wird die Untersuchung auf verschiedenen Korpora basieren. Herangezogen wird vor allem das Korpus FRAN (Français d'Amérique du Nord), das nicht nur eine einzelne französischsprachige Region in Nordamerika berücksichtigt, sondern sich zum Ziel gesetzt hat, das Französische in ganz Nordamerika zu erfassen. Darüber hinaus sollen das Pressekorpus Varitext und das CFPQ (Corpus de français parlé au Québec) in die Überlegungen einbezogen werden, um ein möglichst breites Bild zu erhalten, das verschiedene Textsorten und auch mündliche Quellen umfasst.

\section{Das Wort astheure}

\subsection{Etymologie}

Das Adverb astheure geht nicht direkt auf ein einzelnes lateinisches Wort zurück, sondern ist das Ergebnis der Univerbierung des Ausdrucks à cette heure 'zu diesem Zeitpunkt', einem diachronen Prozess, bei dem mehrere Wörter zu einem einzigen, neuen Wort verschmelzen. Dieser Ausdruck hat laut dem Dictionnaire des régionalismes des îles de la Madeleine (DRIM) mit ad istam oram einen lateinischen Vorläufer (cf. DRIM, s.v. astheure). Das Adverb astheure existierte in dieser Schreibweise bereits im Mittelfranzösischen und ist laut Französischem Etymologischem Wörterbuch (FEW) erstmals für das Jahr 1530 belegt, der Ausdruck à cette heure hingegen schon seit dem Ende des 15. Jahrhunderts (cf. FEW, s. v. hora). Für die Form astora, die dem ursprünglichen lateinischen Ausdruck lautlich deutlich näher kommt, führt das FEW den ersten Beleg für das Jahr 1517 (cf. FEW, s. v. hora).

Das Lemma astheure kommt bis heute nicht in einer einheitlichen, sondern in vielen verschiedenen Schreibweisen vor ${ }^{4}$ die sich jedoch alle mehr oder weniger stark an der Aussprache [astœe] orientieren. Diese Varianz, die Le bon usage als „souvent

${ }^{4}$ Für eine Auflistung der zahlreichen verschiedenen Schreibweisen cf. FEW, s. v. hora. 
peu satisfaisant[e] " (Grevisse/Goosse 2011, 833) charakterisiert, ist auch bei zahlreichen Schriftstellern, beispielsweise bei Montaigne in seinen Essais (cf. Melançon 2016), Charles Péguy (cf. Grevisse/Goosse 2011, 833) oder Balzac im Père Goriot (cf. DCLF, s. v. heure) zu beobachten. Die Ursprungsform à cette heure scheint im Laufe der Zeit von astheure abgelöst worden zu sein, da sie kaum noch Verwendung fand (zwar wurde sie noch von Victor Hugo gebraucht, dies ist aber wohl eher dem Metrum geschuldet, cf. Grevisse/Goose 2011, 833). In allen Fällen der Univerbierung verstummt das $[\varepsilon]$ der ursprünglichen Form cette. Heutzutage scheint sich die Schreibweise astheure und mit Abstrichen auch à $c^{\prime} t^{\prime}$ heure gegenüber anderen historischen Schreibweisen durchgesetzt zu haben, was auch anhand von Wörterbucheinträgen belegt werden kann.

Schließlich hat astheure auch in die französischen Kreolsprachen Amerikas Eingang gefunden, wie es unter anderem Annegret Bollée et al. im Dictionnaire étymologique des créoles français d'Amérique (DECFA) mit dem Vorkommen von astor im Haiti-Kreol für das Jahr 1793 belegen (cf. DECFA, s. v. heure). ${ }^{5}$

\subsection{Wörterbucheinträge}

Natürlich ist nicht nur in etymologischen, sondern auch in zahlreichen weiteren Wörterbüchern ein Eintrag zu astheure wiederzufinden. Im Folgenden sollen nun diese Wörterbucheinträge genauer analysiert werden. Dabei werden zwei Kategorien unterschieden: Wörterbucheinträge unter dem Stichwort astheure oder einer ähnlichen Schreibweise und Wörterbucheinträge, die unter dem Stichwort heure einen Eintrag zu à cette heure vorgenommen haben, in dem sie auch auf astheure eingehen. Berücksichtigt werden bei diesen Überlegungen sowohl allgemeine französische Wörterbücher als auch Wörterbücher, die sich auf das Französische in Nordamerika spezialisiert haben.

Zunächst soll der Fokus auf Wörterbüchern liegen, in denen astheure unter dem Stichwort heure behandelt wird. Alle Einträge dieser Art beginnen mit der Ursprungsform à cette heure und leiten aus ihr die Univerbierung astheure ab. Als wohl bekanntestes französischsprachiges Wörterbuch weist auch der Petit Robert einen solchen Eintrag auf, der allerdings recht kurz ist. Der Eintrag führt maintenant und présentement als Synonyme an und bezeichnet die Verwendung von astheure als "vieilli ou région. (Belgique)" (NPR, s. v. heure). Auch der Dictionnaire culturel en langue française (DCLF) charakterisiert die Verwendung von astheure als „[v]ieilli ou rural“ (DCLF, s. v. heure). Der Dictionnaire Hachette langue française (DH) nennt zwar lediglich die Form à cette heure, bezeichnet aber auch sie als veraltet (cf. DH, s. v. heure). Dass astheure auch in Frankreich verwendet wird, zeigt hingegen der Eintrag im Dictionnaire des régionalismes de France (DRF), der die Regionen aufführt, in denen das Adverb noch heute Verwendung findet (insbesondere im

${ }_{5}$ Angesichts der jungen und vielfach noch nicht abgeschlossenen Normierungsdiskussion im Falle der Kreolsprachen überrascht es kaum, dass das Wort auch dort viele Schreibvarianten zeigt, cf. dazu DECFA, s. v. heure und Thibault $(2015,442)$. 
Norden und Westen Frankreichs). Außerdem wird dort neben Synonymen und zahlreichen Beispielsätzen auch ein Hinweis zur Verwendung gegeben, nämlich dass es populärsprachlich und ländlich verwendet würde (cf. DRF, s. v. heure).

Es gibt jedoch auch zahlreiche Wörterbücher, die für astheure einen separaten Eintrag bieten, es also nicht unter dem Stichwort heure führen, wie dies bei Wörterbüchern zum hexagonalen Französisch und dessen regionalen Varietäten der Fall ist. Dabei handelt es sich jedoch ausschließlich um Wörterbücher zum nordamerikanischen oder noch spezifischer zum kanadischen Französisch. Hierzu zählt beispielsweise der Dictionnaire des régionalismes du français de Terre-Neuve (DRFT) mit dem Lemma astheure, steure, versehen mit der Anmerkung, dass die zweite Form selten sei (cf. DRFT, s. v. astheure, steure). Der Eintrag beinhaltet darüber hinaus Hinweise zum Ursprung des Wortes, Beispielsätze, Literaturangaben und Informationen zum Gebrauch. So wird es als "populaire, vieilli ou régional“, aber auch als "rural" (DRFT, s. v. astheure, steure) angesehen. Ebenso ausführlich ist der Eintrag im Dictionnaire des régionalismes des îles de la Madeleine, der neben Synonymen, der lateinischen Ausgangsform und Beispielen auch auf andere Schreibweisen verweist (cf. DRIM, s. v. astheure). Sogar drei ausführlichere Artikel enthält das Glossaire du parler français au Canada (GPFC) der Société du parler français au Canada zu à c't'heure, asthure und zu astheure (cf. GPFC, s. v. à $c^{\prime} t^{\prime}$ heure, asthure $u$. astheure). Insbesondere letztgenannter ist recht umfassend und enthält neben Synonymen und Beispielen auch Angaben zur Verbreitung in Frankreich, allerdings wird keine Aussage zur Verwendung des Adverbs getroffen. Auch der Eintrag im Dictionnaire québécois-français (DQF) von Meney weist auf die Verwendung von astheure in Nordamerika, Wallonien und einigen ländlichen Regionen Frankreichs hin (cf. DQF, s. v. astheure).

Lediglich knappe Einträge, die astheure teilweise als umgangssprachlich erachten und bei denen zum Teil nur Synonyme und ein Beispielsatz für das Adverb aufgeführt werden, finden sich unter anderem im Dictionnaire général de la langue française au Canada (DLFC, s. v. astheure), im Dictionnaire de la langue québécoise (DLQ, s. v. astheure, asthure), im Dictionnaire québécois d'aujourd'hui (DQA, s. v. ast(h)eure ou à c't'heure), im Dictionary of the Cajun language (DCL, s. v. à $c^{\prime} t^{\prime}$ heure), im Dictionnaire des canadianismes (DC, s. v. à c't'heure u. astheure), im NTC's dictionary of Canadian French (DCF, s. v. astheur, astheure, à c't'heure) und im Dictionnaire de la langue québécoise rurale (DLQR, s.v. astheure (à c't'heure)). Obwohl diese Einträge zum Teil nur sehr kurz sind, zeigen sie, dass die Verfasser das Wort astheure als eigenständig betrachten. Dennoch ist in den meisten dieser Wörterbücher auch unter dem Stichwort heure zusätzlich ein Hinweis zu astheure zu finden. Die Wörterbücher hingegen, die astheure lediglich unter dem Stichwort heure führen, orientieren sich noch stark an der Ursprungsform à cette heure und betrachten astheure offenbar lediglich als ein festes Syntagma mit heure.

In einigen konsultierten Wörterbüchern findet sich jedoch überhaupt kein Eintrag zu astheure, weder als eigenständiger Eintrag noch unter dem Stichwort heure. Dies ist bei den Regionalwörterbüchern von Claude Poirier (Dictionnaire du français québécois (DFQ), Dictionnaire historique du français québécois (DHFQ)) und Normand Beauchemin (Dictionnaire d'expressions figurées en français parlé du Québec 
(DEFFQ)) der Fall, die sich alle auf das Französische in Québec beziehen. Diese Nichtberücksichtigung kann allerdings auch darauf zurückzuführen sein, dass die genannten Wörterbücher keinen Anspruch auf Vollständigkeit erheben und daher nicht alle für das Französische Québecs typischen Wörter berücksichtigen konnten.

Insgesamt ist festzustellen, dass vier der 15 untersuchten Wörterbücher astheure unter dem Stichwort heure erwähnen, wohingegen elf einen eigenen Eintrag zu astheure verfasst haben. Allerdings muss bei dieser Gegenüberstellung bedacht werden, dass astheure lediglich in den Wörterbüchern, die sich auf das Französische Nordamerikas spezialisiert haben, unter einem eigenständigen Eintrag verzeichnet ist, wohingegen es in den großen französischen Wörterbüchern aus Frankreich unter heure abgehandelt wird. Dieser Gedanke soll später nochmals aufgegriffen werden, wenn es um die Verbreitung von astheure gehen wird. Dass sich in Wörterbüchern zum nordamerikanischen Französisch ein separater Eintrag für astheure findet, weist darauf hin, dass es dort im Gegensatz zum hexagonalen Französisch als eigenständiges Lemma angesehen wird, das nicht mehr direkt auf seinen Ursprung heure zurückgeführt wird, weshalb hier auch von Lexikalisierung gesprochen werden kann.

Außerdem führen die Wörterbucheinträge verschiedene Synonyme von astheure an, beispielsweise maintenant, présentement, à présent, dorénavant oder actuellement, die Wörterbücher mit eigenem Eintrag für astheure teilweise auch noch à cette heure. Mit maintenant wird später eines dieser Synonyme hinsichtlich seiner Verwendung in Nordamerika im Vergleich zu astheure untersucht. Dabei wird maintenant für den Vergleich herangezogen, da zum einen die Korpusabfragen in den Korpora FRAN und CFPQ eine ähnlich hohe Frequenz wie für astheure erkennen lassen, zum anderen aber auch die Wörterbücher maintenant häufig als Synonym für astheure anführen und es daher für einen Vergleich geeignet scheint. Im Folgenden muss ferner der Frage nachgegangen werden, inwiefern die unterschiedlichen Gebrauchsmarkierungen (familier, régional, vieilli, populaire), die die Wörterbucheinträge astheure beimessen, tatsächlich zutreffend sind.

\section{Die Verbreitung von astheure}

Der Wortschatz des Französischen Nordamerikas lässt sich grob in fünf Kategorien untergliedern: Archaismen, Dialektalismen, Amerindianismen, Anglizismen und Innovationen (cf. Poirier 1995, 38-42; Martucci 1988, 102-108). Dabei ist das Wort astheure den Archaismen zuzuordnen (cf. Poirier 1995, 38) und damit älterem Sprachgut, das aufgrund der langen Unterbrechung des Kontakts und somit der Abgrenzung zu Frankreich in Nordamerika üblich ist, in Frankreich jedoch kaum oder nicht mehr benutzt wird (cf. Pöll 2017, 92). Astheure ist dabei der kleinen Gruppe an Archaismen zuzurechnen, die sich nicht auf den schriftlich-distanzsprachlichen Bereich beziehen, sondern die der mündlichen Nähesprache zuzuordnen sind, wie der Aufsatz später darlegen wird. Allerdings gilt hier anzumerken, dass eine scharfe Abgrenzung von Archaismen und Regionalismen häufig nicht möglich ist. Ähnlich wie bei astheure handelt es sich bei Archaismen oft um regional spezifische Wörter, die aus regionaler Perspektive keine archaische Konnotation 
besitzen. Nur dort, wo sie außer Gebrauch gekommen sind, gelten sie als vieilli und damit als archaisch.

In der bisherigen Forschung scheint nicht vollständig klar zu sein, inwiefern astheure auch noch in Frankreich verwendet wird. Remysen $(2003,32)$ ist der Meinung, dass astheure im Französischen Frankreichs nicht mehr gebräuchlich sei. Diese Ansicht wurde unter anderem von Avanzi (2019) und Rézeau (DRF, s. v. heure) widerlegt. Darauf aufbauend soll im Folgenden genauer untersucht werden, in welchen Regionen Frankreichs und Nordamerikas astheure heute verwendet wird.

\subsection{Frankreich}

Dass das Adverb astheure in einigen Regionalwörterbüchern, die sich auf die Regionen Frankreichs beziehen, geführt wird, lässt den Schluss zu, dass es sich bei der Verbreitung von astheure in Frankreich nicht um eine flächendeckende, sondern lediglich um eine regionale handelt. Bezüglich der Verbreitung von astheure im europäischen Französisch wurde von Mathieu Avanzi 2017 eine Umfrage durchgeführt, bei der 1376 der 7300 Teilnehmer, die den Großteil ihrer Jugend in Belgien, Frankreich oder der Schweiz verbracht haben, die Frage „Dans la vie de tous les jours, employez-vous le mot astheure au sens de maintenant, à présent ?" bejahten (cf. Avanzi 2019). Die kartographische Darstellung der Ergebnisse (Abb. 1) zeigt für Frankreich, dass astheure vor allem im Poitou, im Aunis, in der Normandie, im Artois und in der Picardie bis heute gebraucht wird, wohingegen es im Süden überhaupt keine Verwendung findet:

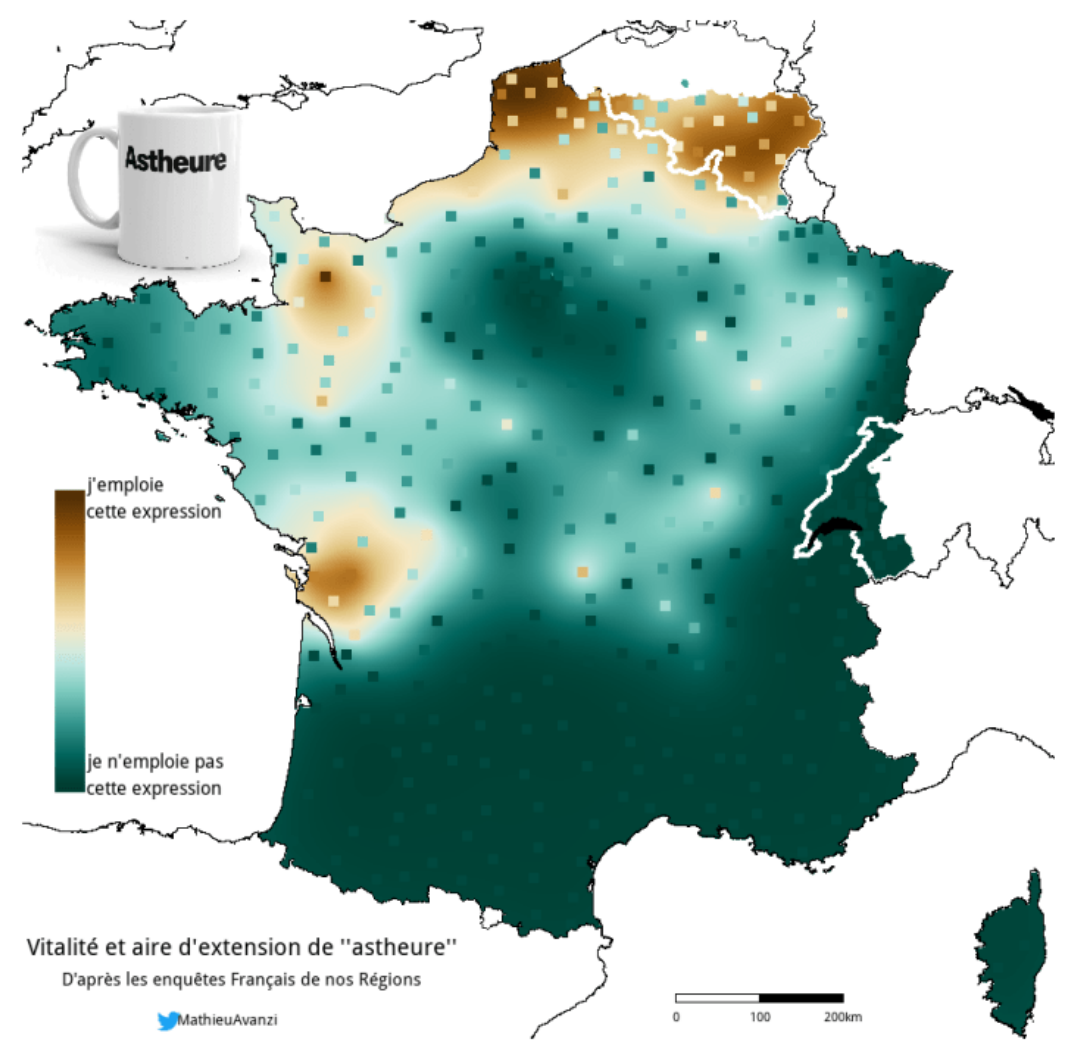

1 | Verwendung des Adverbs astheure in Frankreich, Belgien und der Schweiz, entnommen aus: Avanzi 2019 
Die Erkenntnisse von Avanzis Umfrage decken sich auch mit den Ergebnissen von Rézeau, der "Yvelines ${ }^{6}$, Nord, Pas-de-Calais, Picardie, Normandie, Indre-et-Loire, Bretagne, Mayenne, Sarthe, Maine-et-Loire, Eure-et-Loir, Loir-et-Cher, Champagne, Ardennes, Lorraine" (DRF, s. v. heure) als die Regionen benennt, in denen astheure verwendet wird. Des Weiteren wurde von Avanzi eine Karte des Atlas Linguistique de la France vom Ende des 19. Jahrhunderts neu aufbereitet, um eine eventuell auftretende Veränderung der Verbreitung von astheure erkennen zu können. Bei der damaligen Studie wurden die Befragten gebeten, das Wort maintenant in ihren Dialekt zu übersetzen (cf. Avanzi 2019).

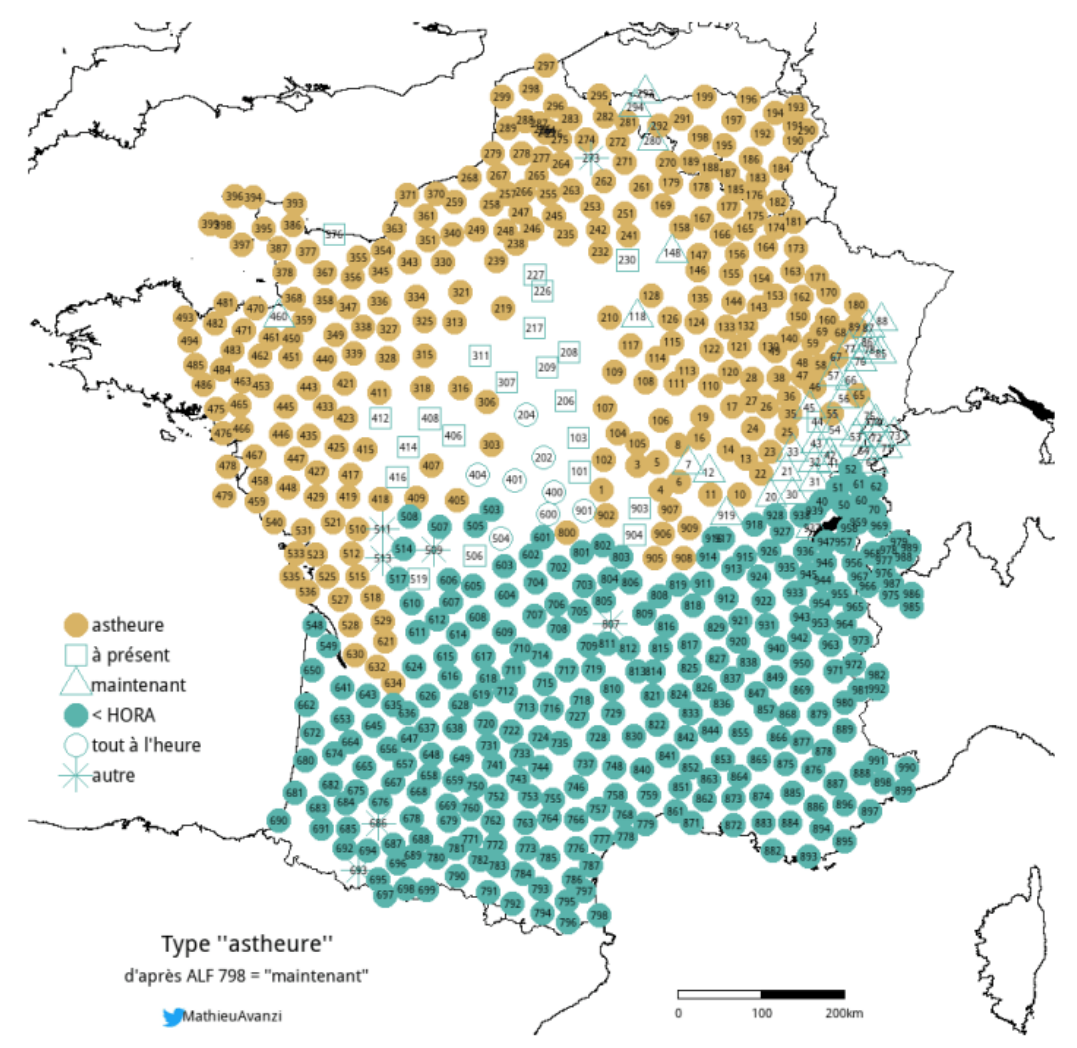

2 | Übersetzungen für maintenant aus den Dialekten des Galloromanischen Ende des 19. Jh., entnommen aus: Avanzi 2019

Die Karte (Abb. 2) zeigt, dass astheure im nördlichen Teil Frankreichs häufig als Entsprechung für maintenant gewählt wurde und daher am Ende des 19. Jahrhunderts in Frankreich noch deutlich weiter verbreitet war als heute. Sie zeigt dabei auch eine Zweiteilung der Sprachsituation in Frankreich ähnlich der Abgrenzung der langues d'oil von den langues d'oc und dem Frankoprovenzalischen (cf. Avanzi 2019). Daher lässt sich festhalten, dass astheure am Ende des 19. Jahrhunderts mit Ausnahme des Zentrums und des äußersten Westens beinahe im gesamten nördlichen Teil Frankreichs verbreitet war, wohingegen es im Süden nicht verwendet wurde. Diese ehemals starke Verbreitung zum Ende des 19. Jahrhunderts lässt im

\footnotetext{
${ }^{6}$ Dass astheure auch im Département Yvelines verwendet wird, bestätigt auch Marie-Rose Simoni-Aurembou
} $(2004,64)$. 
Vergleich mit den aktuellen Daten von 2017 außerdem darauf schließen, dass die Verwendung von astheure in Frankreich nachlässt. Auch die Tatsache, dass astheure in den großen französischen Wörterbüchern unter keinem eigenen Eintrag, sondern lediglich unter dem Stichwort heure geführt wird, verdeutlicht nochmals, dass es sich bei diesem Adverb in Frankreich lediglich um eine regionale Variante handelt.

Darüber hinaus fällt bei den Karten zur Verbreitung von astheure auf, dass im nördlichen Teil Frankreichs insbesondere die Île de France eine Region zu sein scheint, in der astheure überhaupt nicht gebraucht wird. Es ist zu vermuten, dass auch die Negativbewertung bzw. Nichtberücksichtigung von astheure in der sprachkritischen Tradition des bon usage und der Académie française des 17. und 18. Jahrhunderts, die Regionalismen ohnehin ablehnend gegenüberstand (cf. Mazière 1995, 15; Morvan 2019, 298-299), zu einem Rückgang seiner Verbreitung führte. Diese Tradition orientierte sich stark am Pariser Französisch, dem français de référence, und privilegierte damit bestimmte Wörter bzw. lehnte wiederum andere ab. Ein solches Vorgehen entsprach allerdings nicht zwangsläufig den Sprechgewohnheiten der Zeit. So führt keines der Akademiewörterbücher die Form astheure, obwohl das Wort zur Zeit der ersten Auflage bereits in Gebrauch war. Die Wörterbücher betrachten lediglich à cette heure als festes Syntagma, welches sie unter dem Stichwort heure führen. Während die Erstauflage des Akademiewörterbuchs (DAF1) 1694 noch keine Negativbewertung dieses Syntagmas vornahm (cf. DAF1, s. v. heure), wurde à cette heure in der dritten Auflage (DAF3) im Jahr 1740 durch den Hinweis familier bereits mit einer negativen Konnotation versehen (cf. DAF3, s. v. heure). So dürfte auch die Negativbewertung des Ausdrucks durch die Académie française, die sich stark am Pariser Französisch orientierte, in dem astheure nicht verwendet wurde, zu einem Rückgang seiner Verwendung geführt haben.

\subsection{Nordamerika}

Anders stellt sich die Situation in den französischsprachigen Gebieten Nordamerikas dar. Hier nämlich ist das Adverb astheure flächendeckend verbreitet. In Québec beispielsweise erfreut es sich bis heute großer Beliebtheit, wie eine Karte von Avanzi (2019) zeigt, die auf Erkenntnissen des Atlas Linguistique de l'Est du Canada basiert, der in den 1970 er Jahren durch Befragungen jedoch vornehmlich älterer und ländlicher Bevölkerungsgruppen erarbeitet und 1980 veröffentlicht wurde: 


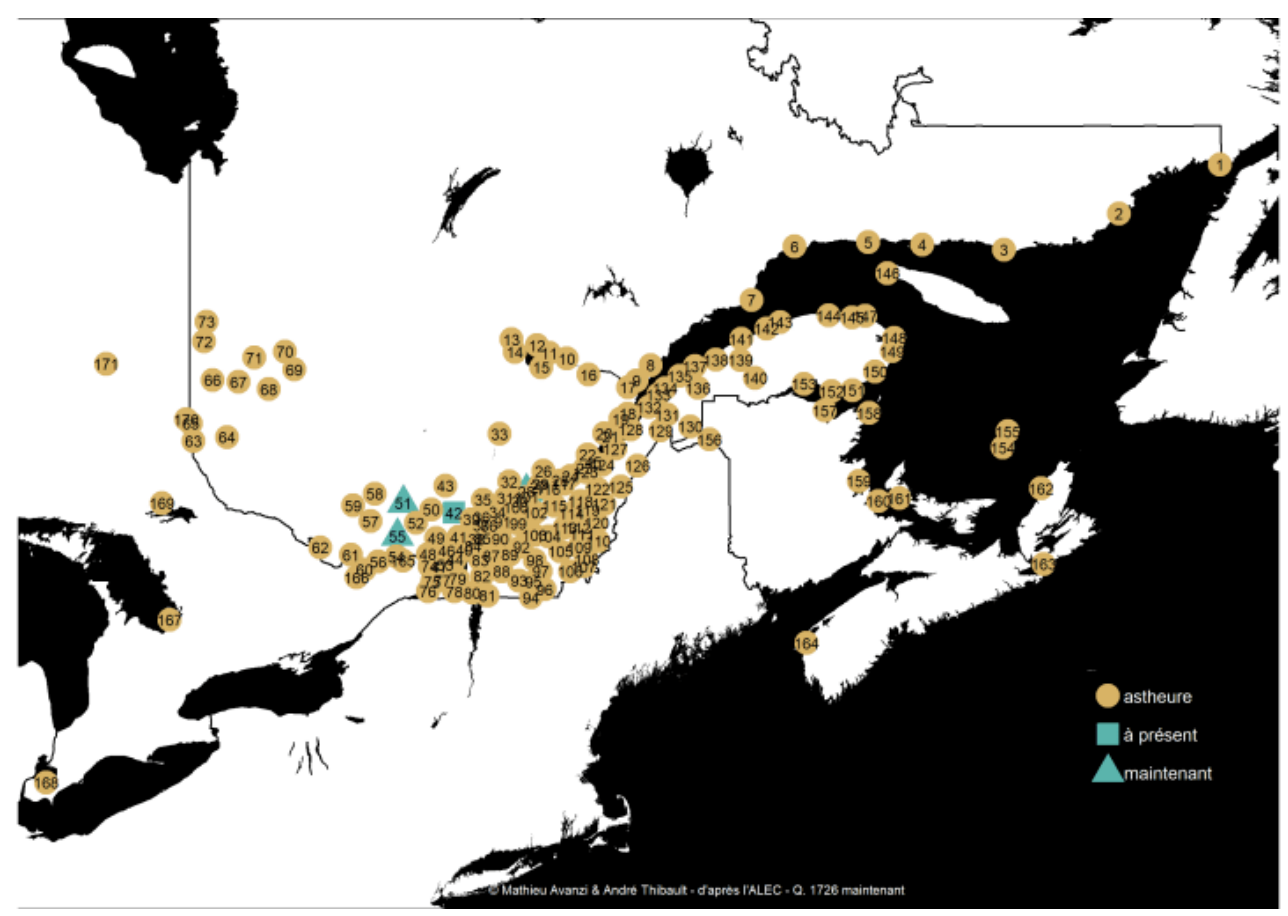

3 | Die Verwendung von astheure und seiner Synonyme maintenant und à présent in Kanada in den 1970er Jahren, entnommen aus: Avanzi 2019

Nach dieser Karte (Abb. 3) war astheure in Kanada in den 1970er Jahren sehr weit verbreitet, während Synonyme wie maintenant, das lediglich dreimal von den Befragten als bevorzugte Variante angegeben wurde, und à présent (einmal) im Vergleich zu astheure kaum Verwendung fanden. Vor allem in Québec scheint astheure gebräuchlich zu sein, es wird aber auch in der Akadie und in Teilen Ontarios verwendet, wo es im Vergleich zu maintenant und à présent ebenfalls das bevorzugte Adverb der Sprecher zu sein scheint. Diese Tatsache lässt sich auch auf Louisiana übertragen (cf. Avanzi 2019), das auf der Karte allerdings nicht dargestellt ist. Dass Avanzis Annahme, wonach astheure auch in Louisiana gebräuchlich sei, aber durchaus zutrifft, zeigt Stäbler (1995a, 247), die das Adverb unter der Schreibweise asteur auch in ihrem Glossar zum cadien führt. Auch Bergeron-Maguire (2011, 65-66) weist auf seine Verwendung in Louisiana hin, merkt allerdings an, dass im cadien eher die Schreibweise asteure üblich sei. Dass astheure neben Québec auch in Louisiana, Ontario und der Akadie gebräuchlich ist, zeigt ferner ein erster Blick auf das Korpus FRAN, das für astheure Belege aus allen diesen Regionen führt.

Auch eine bei Louis Mercier $(2002,299)$ angeführte Studie, die allerdings bereits zu Beginn des 20. Jahrhunderts durchgeführt wurde, kommt zu dem Schluss, dass astheure damals von $98 \%$ der französischsprachigen Bevölkerung Kanadas verwendet wurde.

Doch bis heute scheint astheure in Québec noch sehr gebräuchlich zu sein, wie die Lokalisierung der Belege für astheure aus einem von Avanzi (2019) erstellten Twitter-Korpus zeigt: 


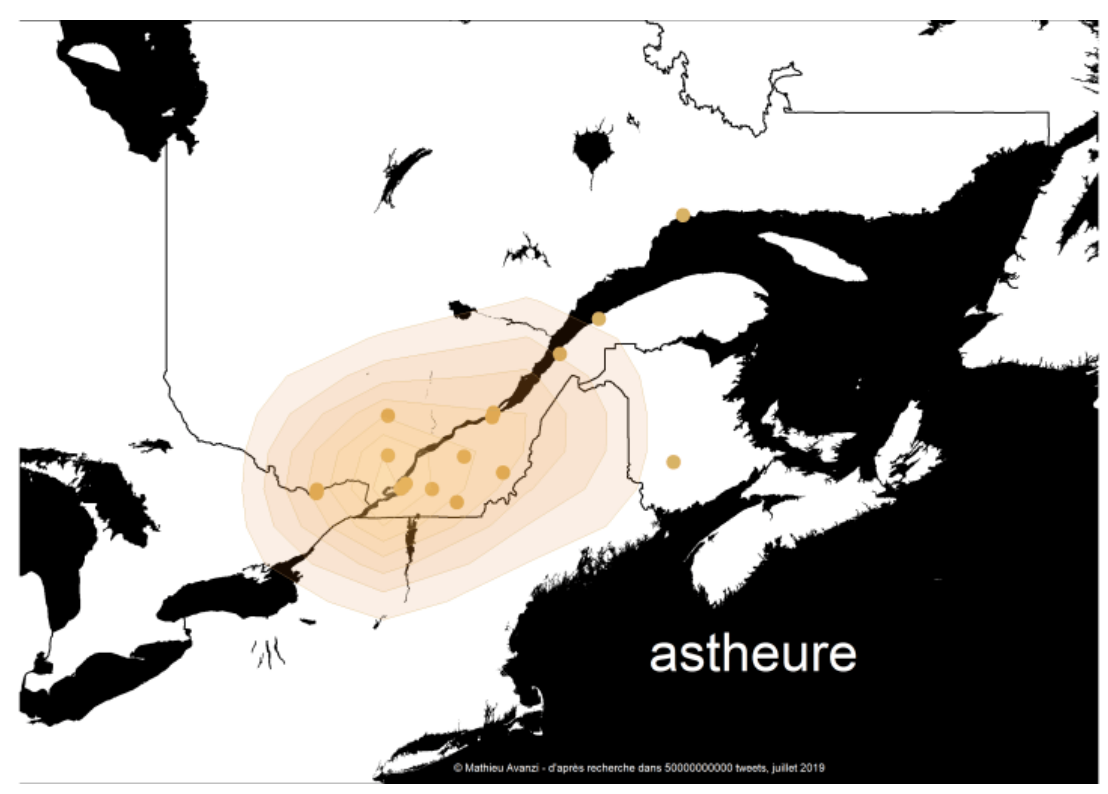

4 | Verbreitung von astheure in Kanada im Jahr 2019, entnommen aus: Avanzi 2019

Die Karte (Abb. 4) belegt, dass astheure in Tweets im ganzen französischsprachigen Teil Kanadas verwendet wird und nicht nur auf Québec beschränkt ist, wenngleich ein Großteil der Tweets von dort ansässigen Personen stammt. Demgegenüber konnte eine Verwendung von astheure im europäischen Twitter-Französisch nicht nachgewiesen werden. Dies muss jedoch nicht bedeuten, dass astheure dort keine Verwendung findet, sondern wohl in anderen Kommunikationskontexten gebraucht wird (cf. Avanzi 2019).

So stellt sich nun die Frage, warum astheure in Nordamerika flächendeckend gebräuchlich ist, wohingegen das Adverb in Frankreich lediglich als Regionalismus angesehen werden kann. Zur Klärung dieser Frage kann ein Blick auf die Auswanderer nützlich sein, die Frankreich im 17. und 18. Jahrhundert in Richtung Nordamerika verlassen haben.

\begin{tabular}{|c|c|c|c|}
\hline & 17. Jhdt. & & 18. Jhdt. \\
\hline Normandie $^{54}$ & $18.5 \%$ & île-de-France & $12,2 \%$ \\
\hline Île-de-France/Paris & $14,7 \%$ & Normandie & $10,9 \%$ \\
\hline Poitou & $10,9 \%$ & Bretagne & $8,2 \%$ \\
\hline Aunis, lle de Ré, lle d'Oléron & $10,6 \%$ & Poitou & $6,0 \%$ \\
\hline Saintonge & $5,8 \%$ & Guyenne, Agenois & $5,8 \%$ \\
\hline Perche & $3,9 \%$ & Saintonge & $5,5 \%$ \\
\hline Bretagne & $3,5 \%$ & Aunis, Île de Ré & $5,6 \%$ \\
\hline Anjou & $3,0 \%$ & Languedoc & $5,2 \%$ \\
\hline Champagne & $2,8 \%$ & Gascogne & $4,6 \%$ \\
\hline Maine & $2,7 \%$ & Champagne & $3,4 \%$ \\
\hline Guyenne & $2,6 \%$ & Lorraine & $2,6 \%$ \\
\hline Limousin, Périgord & $2,4 \%$ & Anjou & $2,6 \%$ \\
\hline Picardie & $2,2 \%$ & Franche-Comté & $2,1 \%$ \\
\hline Angoumois & $2,0 \%$ & Picardie & $2,2 \%$ \\
\hline Touraine & $1,9 \%$ & Bourgogne & $2,1 \%$ \\
\hline Beauce & $1,9 \%$ & & \\
\hline
\end{tabular}

Tab. 1 |Übersicht zur Herkunft der französischstämmigen Siedler in Kanada im 17. und 18. Jahrhundert, entnommen aus: Pöll $(2017,86)$ 
Der Tabelle (Tab. 1) nach stammten die französischen Siedler, die sich im 17. und 18. Jahrhundert in Kanada niederließen, vor allem aus dem Norden, besonders der Normandie, und dem Westen, wohingegen kaum Personen aus dem Süden nach Kanada übersiedelten. Pöll $(2017,86)$ gibt allerdings an, dass die neuere Forschung eher der Meinung sei, dass zumindest von 1608 bis 1680 der größte Anteil der Siedler aus dem Westen stammte. Nichtsdestotrotz machten auch in diesem Zeitraum die Siedler aus dem Norden Frankreichs einen großen Anteil der Auswanderer aus.

Die Herkunft der Siedler anhand einer Karte dargestellt zeigt dann Folgendes:

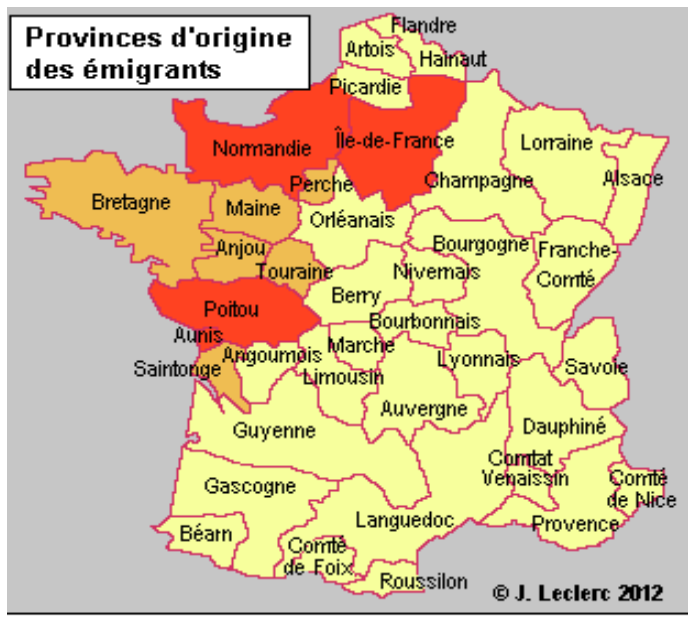

Provinces numériquement prépondérantes

5 | Herkunft der französischstämmigen Siedler in Kanada, entnommen aus: Leclerc 2017

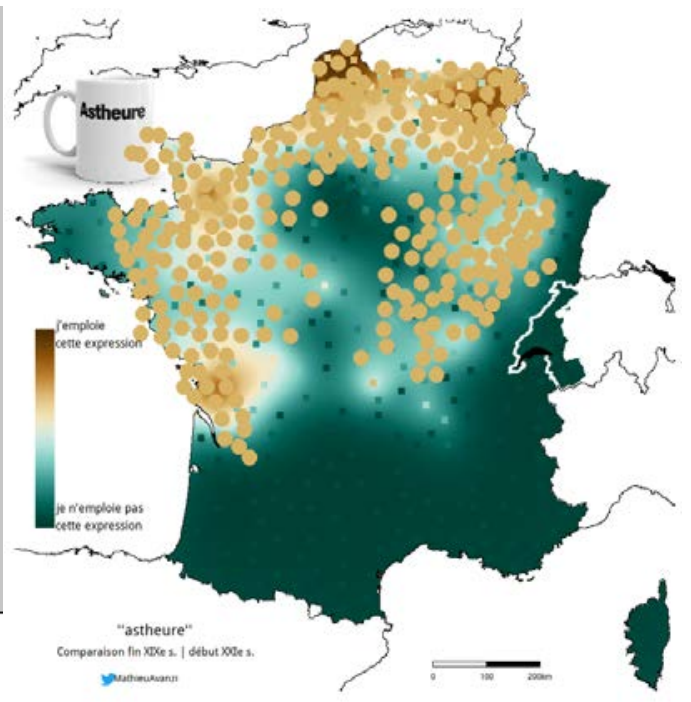

6 | Vergleich der Verwendung von astheure am Ende des 19. und zu Beginn des 21.

Jahrhunderts, entnommen aus: Avanzi 2019

Vergleicht man nun die Regionen, aus denen die französischen Siedler stammten, die sich in Nordamerika niederließen (Abb. 5), mit einer Karte (Abb. 6), die Abb. 1 und Abb. 2 kombiniert, so stellt man fest, dass die Siedler überwiegend aus den Regionen kamen, in denen astheure im 19. Jahrhundert und bis heute verwendet wird. Daher ist davon auszugehen, dass dieses Wort in diesen Regionen auch bereits zur Zeit der Auswanderung der Siedler im Gebrauch war. Viele von innen stammten aus dem Aunis, dem Poitou und der Normandie, wo astheure bis heute stark vertreten ist. Daher ist zu vermuten, dass dort astheure seit Generationen stark im Sprachgebrauch verankert war und von den Siedlern in Nordamerika implantiert wurde. Auch für André Thibault gehört astheure zu einer Gruppe von Wörtern, "qui devaient pourtant être très fréquents dans le français vernaculaire des colons des XVII -XVIII e siècles" (Thibault 2016, 75). Wäre dagegen der Großteil der Siedler aus dem Süden Frankreichs gekommen, so wäre zu vermuten, dass dann heutzutage in Nordamerika ein anderes auf das lateinische hora zurückgehendes Synonym von maintenant aus dem Okzitanischen (bspw. ara) auch in Nordamerika verwendet würde (s. dazu auch Abb. 2) - so hat sich allerdings astheure durchgesetzt. Aufgrund der mit der britischen Herrschaft ab 1763 einsetzenden politischen Trennung und der damit einhergehenden langen Unterbrechung des Kontakts zu Frankreich blieb in Nordamerika der langsame Rückgang des Gebrauchs 
von astheure aus. Daher wird das Adverb astheure bis heute in Nordamerika flächendeckend verwendet.

\section{Astheure und maintenant in Nordamerika im Vergleich}

Für den nun folgenden Vergleich der beiden Temporaladverbien astheure und maintenant im Hinblick auf ihre Verwendung und Bedeutung wurde letzteres ausgewählt, da es im Gegensatz zu anderen Synonymen wie à présent oder dorénavant das einzige zu sein scheint, das eine ähnlich häufige Verwendung wie astheure aufweist. $^{7}$

Als Basis für die Gegenüberstellung dienen Korpusabfragen, die sich allerdings auf das nordamerikanische Französisch beschränken, da astheure darin wie bereits gesehen wesentlich gebräuchlicher ist. Um möglichst viele verschiedene Verwendungsweisen von astheure und maintenant aufzuzeigen und Ergebnisse zu verschiedenen Textsorten sowie auch zu mündlichem Sprachgebrauch zu erhalten, wurden drei Korpora zur Untersuchung herangezogen. Die Wahl fiel zuerst auf das Korpus FRAN (Français d'Amérique du Nord), das Texte aus dem gesamten französischsprachigen Teil Nordamerikas vom 18. bis ins 21. Jahrhundert enthält, und zwar vor allem persönliche Briefe und Gespräche. Außerdem kamen Varitext, ein Korpus kanadischer Pressetexte, ${ }^{8}$ und das CFPQ (Corpus de français parlé au Québec), ein Korpus für spontane und freie Konversationen, zum Einsatz. ${ }^{9}$

\subsection{Korpusuntersuchung zur Verwendung von astheure}

Einen ersten Eindruck von der Verwendung von astheure gibt die folgende Übersicht über seine Vorkommenshäufigkeit in den jeweiligen Korpora. Dabei wurden alle bekannten Schreibweisen des Lemmas astheure berücksichtigt, der Einfachheit halber aber unter der Graphie astheure zusammengefasst. ${ }^{10}$

\begin{tabular}{|l|l|l|}
\hline Korpus & Anzahl Treffer & Prozentualer Anteil am Korpus \\
\hline FRAN & 1728 & $0,144 \%$ \\
\hline Varitext & 4 & $0,0000075 \%$ \\
\hline CFPQ & 103 & $0,015 \%$ \\
\hline
\end{tabular}

Tab. 2 | Statistische Übersicht zum Vorkommen von astheure in den verwendeten Korpora

\footnotetext{
7 Dies zeigt bereits ein erster Blick auf das Korpus FRAN, bei dem man für Synonyme wie à présent (13 Treffer), présentement (86 Treffer), à cette heure (5 Treffer), dorénavant (1 Treffer), désormais (0 Treffer) oder actuellement (79 Treffer) deutlich weniger Treffer erhält als für astheure (1728 Treffer) und maintenant (1523 Treffer).

8 Das Pressekorpus Varitext enthält Pressetexte aus großen Teilen der Frankophonie. Für die vorliegende Untersuchung wurden jedoch nur kanadische Pressetexte berücksichtigt, indem für die Abfragen ein Subkorpus mit ausschließlich kanadischen Pressetexten erstellt wurde.

9 Alle verwendeten Korpora sind online abrufbar. Die jeweilige URL ist dem Kap. 6 Quellen- und Literaturverzeichnis zu entnehmen.

${ }^{10}$ Wenn auch im Folgenden von astheure die Rede sein wird, so bezieht sich der Aufsatz immer auf alle das Lemma umfassenden Schreibweisen. Abgefragt wurden die Graphien astheure, astheur, asteure, asteur und à c't'heure.
} 
Es fällt auf, dass das Adverb astheure im Korpus FRAN mit 1728 Belegen am häufigsten vorkommt, auch prozentual gesehen. Auch im CFPQ ist es mit 103 Treffern noch recht häufig, im Pressekorpus Varitext hingegen kommt das Wort insgesamt lediglich viermal vor und hat dadurch einen äußerst geringen Anteil am gesamten Korpus.

Die Wörterbucheinträge haben gezeigt, dass bei astheure häufig auf dessen umgangssprachliche Verwendung hingewiesen wird, weshalb dieser Aspekt im Folgenden besonders starke Berücksichtigung findet. Darüber hinaus wird aber auch darauf eingegangen, mit welchen Verben und mit welchem Tempus astheure gebraucht wird, was bei Temporaladverbien besonders aufschlussreich zu sein scheint. Ferner wird die Position des Adverbs im Satz thematisiert.

Zunächst geht es darum, in welchen Varietäten astheure verwendet wird. Im Korpus FRAN ist festzustellen, dass lediglich 16 aller Belege für astheure aus Jahren vor 1976 stammen, die alle auf Privatbriefe entfallen. Die restlichen über 1700 Belege stammen alle aus der Zeit danach, und zwar aus den zahlreichen ab 1976 aufgenommenen privaten Gesprächen, also aus nähesprachlichem mündlichen Sprachgebrauch. Demzufolge ist astheure dem français familier zuzuordnen, was Langlois $(1999,90)$ bestätigt, die astheure bei der Auflistung der Wörter des Registers oral très familier an neunter Stelle führt. Sie stützt sich auf eine Umfrage, bei der die Teilnehmer darum gebeten wurden, Wörter anhand ihrer Verwendung zu klassifizieren. Dabei wurde astheure von gut zwei Drittel der Befragten als oral familier (40 Nennungen) oder als oral très familier (55 Nennungen) eingestuft, wobei zwölf der 146 Teilnehmer astheure gar als vulgären Ausdruck ansahen (cf. Langlois, 44). Für die Frage nach der variationellen Einordnung von astheure lohnt sich ebenso ein Blick auf das Pressekorpus Varitext, in dem astheure insgesamt viermal belegt ist:

AS1:

"T'as volé pour moi, j'te serre la main, c'est beau. Astheure ferme ta gueule pis fait l'idiot. T'as rien qu'à être d'accord, moi j'mets du vin d'ins pots. [...] » (Korpus Varitext)

AS2:

[C'est] comme si les érables pissaient astheure de l'huile à moteur. (Varitext)

AS3:

Ce qu'on propose " astheure » a beaucoup gagné musicalement. (Varitext)

AS4:

"T'en souviens-tu Godin astheure que t'es député de l'homme qui frissonne en attendant l'autobus de petit matin après son shift de nuit ? 》(Varitext)

AS1 und auch AS4 lassen sich ebenfalls klar der gesprochenen Nähesprache zuordnen, nicht zuletzt, weil astheure in direkter Rede erscheint. Gestützt wird dies außerdem durch für die Nähesprache typische Phänomene wie die Elision der Auslautvokale bei tu und vorkonsonantischem je. Darüber hinaus verweist ferme ta gueule auf ein oral très familier. AS2 stammt aus einer Art Leserbrief. Auch in solchen freieren Textsorten werden teilweise nähesprachliche Formulierungen ver- 
wendet. AS3 kann ganz außer Acht gelassen werden, da es sich bei dieser Verwendung von astheure um den Namen einer Musikgruppe handelt, weshalb es in diesem Zeitungsartikel auch in Anführungszeichen gesetzt wurde. In der kanadischen Presse wird astheure also beinahe nicht verwendet, außer in direkten Zitaten oder in freieren Textformen wie der des Leserbriefs. Allerdings ist es auch dort so selten, dass der fehlende Gebrauch in Pressetexten den Charakter von astheure als oral familier oder gar als oral très familier unterstreicht.

Die mit einem statistischen Kookkurrenzmaß ermittelten Kookkurrenzen von astheure, die mithilfe des Korpus FRAN bestimmt werden können, bestätigen diesen Eindruck. Bei dieser Kookkurrenzabfrage wird ein sogenannter Score errechnet, der eine Aussage über die Wahrscheinlichkeit, dass bestimmte Wörter zusammen auftreten, trifft. Unter den Kookkurrenzen von astheure ist auch ça zu finden, das in der Schriftsprache nicht in dieser verkürzten, sondern in seiner standardsprachlichen Form cela verwendet würde. Auch das bereits erwähnte TwitterKorpus von Avanzi (2019) führt Belege für astheure. Dies ist ein weiteres Indiz für seine Verwendung unter den Bedingungen kommunikativer Nähe und konzeptioneller Mündlichkeit, wie sie elektronische Kurznachrichten in bestimmter Hinsicht prägen (cf. Beißwenger 2016, 22-24; Knaz 2015). Spezifisch für Twitter konnte dabei im Allgemeinen neben der Verwendung diatopischer und diaphasischer Elemente ein enger Zusammenhang der Tweets zur gesprochenen Umgangssprache nachgewiesen werden (cf. Overbeck 2012, 231-232). Dieser variationelle Status der Tweets ist ein weiterer Hinweis auf die mündlich-nähesprachliche Verwendung von astheure, da es wie bereits gesehen auch in Tweets gebraucht wird. Laut Thibault (2009, 111-113) wird astheure in Nordamerika dabei jedoch weniger stark als populärsprachlich angesehen als in Frankreich.

Wenden wir uns nun der Frage zu, mit welchen Verben und mit welchem Tempus astheure verwendet wird. Die große Anzahl an Belegen aus den drei Korpora erforderte die Beschränkung auf eine exemplarische Auswahl von hundert Belegen. Dabei wurde versucht, sowohl ältere als auch neuere Belege zu berücksichtigen, um ein möglichst aussagekräftiges Ergebnis zu erhalten. Diese zufällig ausgewählten hundert Belege orientieren sich proportional an der Gesamtzahl der Belege für astheure aus den drei Korpora. ${ }^{11}$

Unter den mit astheure gebrauchten Verben sind être und avoir die mit Abstand häufigsten, was sich nicht zuletzt durch die zahlreich mit dem Adverb vorkommenden Präsentative c'est und il y a erklärt. ${ }^{12}$ Zum einen sind derartige Konstruktionen für die Nähesprache kennzeichnend (cf. Koch/Oesterreicher 2011, 114-116) und stützen damit die dargelegte diasystematische Situierung von astheure. Zum anderen dienen sie häufig zur Beschreibung eines Zustands zum aktuellen Zeitpunkt, wofür offenbar auch astheure eingesetzt wird. Ferner fällt als Kopulaverb neben dem bereits erwähnten être auch rester (vier Vorkommen mit

11 Bei der zufälligen Auswahl der Belege wurde jeder 18. Treffer aus den Korpora in die Belegliste aufgenommen.

12 Dabei treten c'est und il y $a$ in den hundert ausgewählten Belegen 48 Mal (c'est) bzw. 22 Mal (il y $a$ ) gemeinsam mit astheure auf. 
astheure) auf. Ansonsten lässt die Untersuchung allerdings nicht auf Verben schließen, die bevorzugt mit astheure verwendet werden. Eine diesbezüglich verlässliche Aussage hätte sich auf eine Auswertung einer deutlich größeren Zahl an Sätzen und ihren anschließenden Vergleich mit einer Frequenzliste der häufigsten französischen Verben zu stützen, was den Rahmen dieses Aufsatzes sprengen würde.

Bezüglich des Tempus allerdings ergibt sich ein eindeutiges Resultat: In beinahe allen untersuchten Sätzen wird das présent verwendet, in lediglich sieben Fällen andere Zeitformen. Diese Tatsache verdeutlicht, dass sich astheure kaum auf die Vergangenheit oder die Zukunft bezieht, sondern beinahe immer auf die Gegenwart und somit den Zeitpunkt des Sprechens bzw. des Schreibens (To).

Astheure muss sich aber nicht nur auf die Gegenwart beziehen, sondern kann in gewissen Fällen auch die Zukunft einschließen und auf diese Weise als Synonym zu dorénavant und désormais fungieren (die übrigens beide wie astheure ein auf das lateinische hora zurückgehendes Bildungselement enthalten, cf. Séguin 2011, 138), wie es der folgende Beleg veranschaulicht:

AS5: ${ }^{13}$

a) Astheure, les femmes se laissent pas marcher sur le corps comme avant. (FRAN)

b) Désormais, les femmes se laissent pas marcher sur le corps comme avant.

In Beispiel AS5 scheinen désormais 'von jetzt an' und astheure austauschbar zu sein, weil hier offensichtlich ein Anhalten der Situation über To hinaus nicht auszuschließen ist. Dass dies allerdings nicht immer zutrifft, zeigen AS6 und AS7, in denen désormais und auch dorénavant nicht als Synonyme für astheure dienen können.

AS6:

a) Astheure je le regarde. (FRAN)

b) * Dorénavant je le regarde.

AS7:

a) C'est juste / c'est tous les mêmes qui viennent astheure. (FRAN)

b) * C'est juste / c'est tous les mêmes qui viennent désormais.

In Beispiel AS6 kann dorénavant nicht als Synonym für astheure dienen, da der Vorgang, der in diesem Beispiel beschrieben wird, zeitlich begrenzt ist und die Zukunft nicht einschließt, sondern sich lediglich auf den Zeitpunkt des Sprechens und damit auf die Gegenwart bezieht. Ähnlich wie in Beispiel AS6 beschränkt sich astheure auch in AS7 auf To, was dadurch veranschaulicht werden kann, dass in diesem Fall astheure durch eine konkrete Zeitangabe, beispielsweise à treize heures, ersetzt werden könnte, ferner wie auch in AS6 durch das in fast allen Fällen auf To bezogene maintenant (cf. Bertin 2001, 42).

\footnotetext{
${ }^{13}$ Sind im Folgenden in einem Beispiel zwei Versionen (Version a) und Version b)) angegeben, so stammt Version a) aus einem der Korpora, während in Version b) astheure durch ein Synonym ersetzt wurde, um den Satz auf seine Grammatikalität zu prüfen.
} 
Des Weiteren wird astheure teilweise im Sinne von de nos jours 'heutzutage' gebraucht, wie folgende Beispiele aus den abgefragten Korpora zeigen:

AS8:

[A]stheure si une personne meurt à soixante-cinq "Oh mais elle était assez jeune. Il était assez jeune. " Pas vrai. (FRAN)

AS9:

Moi je crois que c'est / astheure ça c'est un " problem ». (FRAN)

Ansonsten kann astheure verwendet werden, um die Gegenwart und die Vergangenheit einander gegenüberzustellen, wie folgende Beispiele zeigen:

AS10:

Pis là ils ont bâti ce rue-là pis astheure il y a une astheure qui traverse en travers là. (FRAN)

AS11:

Dans ce temps-là tu passais un test avant là euh euh euh c'était pas comme astheure là. (FRAN)

AS10 stellt die mittels des passé composé beschriebene Vergangenheit, nämlich den Zeitpunkt, an dem die Straße gebaut wurde, dem durch das présent verdeutlichten Zeitpunkt des Sprechens To gegenüber, an dem die Straße bereits fertiggestellt und benutzbar ist. Auch AS11 kontrastiert einen Zeitraum aus der Vergangenheit mit $T_{0}$, entsprechend beinhaltet der Satz ein Verb im imparfait.

Die Funktion, die astheure in einem Satz erfüllen kann, beschränkt sich jedoch nicht auf die Temporaldeixis. Wie Vogh $(2018,122)$ zeigen konnte, wird es auch verwendet, um abrupte Themenwechsel zu vollziehen. Diese Erkenntnis kann die vorliegende Korpusuntersuchung bestätigen:

AS12:

Alexandre: Non. Comme cette / cette chanson dit astheure euh "I'll be eighteen 'til I die ».

Tous : (rires)

11: Ou trente-neuf comme Jack Benny.

12: Oui. Toujours trente-neuf.

Alexandre : Astheure j'ai juste des / des / des / "partys » de mon quarante " birthday. That's something else. Birthday in French ». (FRAN)

In dem ersten Wortbeitrag des Sprechers Alexandre besitzt astheure keine temporaldeiktische Funktion, sondern wird als Diskursmarker verwendet und erfüllt damit eine pragmatische Funktion. Es dient hier in gewisser Hinsicht wie auch das nachfolgende euh als Verzögerungssignal ohne semantische Bedeutung. Auch in seinem zweiten Wortbeitrag verwendet der Sprecher das Wort astheure. Hier kommt dem Adverb ebenfalls eine diskursmarkierende Funktion zu. Er nutzt es, um ein neues Thema einzuleiten, das in keinem direkten Zusammenhang zum vorangegangenen 
Thema steht. ${ }^{14}$ Seine Verwendung trägt hier lediglich zur Strukturierung des Beitrags bei und kündigt den Themenwechsel an. Der offenbar bilinguale Sprecher weist sogar selbst darauf hin, dass die Information something else sei. Der Themenwechsel kündigt sich dabei nicht nur durch die Verwendung von astheure und den expliziten Hinweis auf das neue Thema an. Auch das Code-switching macht den Themenwechsel und den neuen Gesprächsabschnitt deutlich, da der Wechsel der Sprache hier auch den Wechsel des Themas anzeigt. ${ }^{15}$

Im Akadischen von Nouveau-Brunswick kann astheure als Synonym von ça fait auch als Sukzessionssignal verwendet werden (cf. Péronnet 1989, 188-189 u. 198). Dass es auch im cadien Louisianas diese Funktion erfüllen kann und nicht, wie Stäbler (1995b, 140) angibt, immer als temporale Angabe gebraucht wird, zeigt sich in diesem Beispiel aus Louisiana:

\section{AS13:}

Ah c'était eusse qui / qui envoyaient la chevrette dans plusieurs parties de les États-Unis ou du / du monde. Et c'était tout mis dans des / dans des barils. Astheure aujourd'hui euh mettont ça dans des / dans des sacs. (FRAN)

Astheure kommt in diesem Beispiel vor allem die Funktion als Sukzessionssignal und nicht jene als temporale Angabe zu, da die Temporaldeixis in diesem Beispiel durch aujourd'hui ausgedrückt wird, das $T_{0}$ der Vergangenheit gegenüberstellt. Astheure wird hier am Satzanfang platziert, um die in den beiden Sätzen übermittelten Informationen aneinanderzureihen und logisch miteinander zu verbinden. Da astheure wie bereits gesehen als Diskursmarker konventionalisiert zu sein scheint, kann es ausgehend von seiner diskursmarkierenden Funktion einen weitergehenden Bedeutungswandel durchlaufen und wie in Beispiel AS13 die Funktion als logischer Satzkonnektor erfüllen. Dabei trägt astheure eine adversative Bedeutung und wird zur Hauptsatzkonjunktion. Auch andere Temporaldeiktika wie alors, or oder das spanische ahora (cf. Matus-Mendoza 2000, 71), die im Übrigen wie astheure ein auf das lateinische hora zurückgehendes Bildungselement enthalten, haben einen solchen Wandelprozess durchlaufen und werden als Hauptsatzkonnektoren mit adversativer Bedeutung verwendet (cf. Frank-Job 2010, 301-302). Während jedoch alors, or oder auch das italienische allora ihre Temporaldeixis zugunsten ihrer diskursmarkierenden Funktion fast völlig abgelegt haben, besitzt astheure neben seiner diskursmarkierenden weiter eine temporaldeiktische Funktion.

Im Hinblick auf die Position von astheure im Satz lässt sich im Korpus FRAN beobachten, dass sich ungefähr die Hälfte aller Belege des Adverbs in der Mitte des Satzes befindet, während gut ein Viertel an erster, ein weiteres Viertel der Fälle an

\footnotetext{
14 Dass die Verwendung von astheure dabei im Allgemeinen häufig mit Verzögerungssignalen oder Pausen einhergeht, deutet darauf hin, dass die Sprecher auf der Suche nach einem neuen Gesprächsthema sind, was wiederum die These, wonach astheure bei abrupten Themenwechseln genutzt wird, stützt (cf. Vogh 2018, 122).

${ }^{15}$ Ausgelöst wird das Code-switching wohl durch die Tatsache, dass dem Sprecher das Wort fête nicht in den Sinn kommt, worauf die mehrmalige Wiederholung des Artikels des hindeutet, und er deshalb auf die englische Entsprechung party zurückgreift. Einzig aus der Verwendung von party erklärt sich jedoch nicht, warum der Sprecher auf Englisch fortfährt, da er seinen Satz zunächst auf Französisch weiterführt (de mon quarante), weshalb das Code-switching hier wahrscheinlich die Markierung des Themenwechsels unterstützt.
} 
letzter Stelle des Satzes steht. Dabei wurde das Korpus FRAN zur Positionsbestimmung herangezogen, da es das einzige der ausgewählten Korpora ist, das eine automatische Positionsbestimmung aller Treffer und damit eine umfassende Untersuchung erlaubt.

\begin{tabular}{|l|l|l|}
\hline Position & Anzahl Treffer & Prozentualer Anteil \\
\hline Satzbeginn & 392 & $22,69 \%$ \\
\hline Satzmitte & 856 & $49,54 \%$ \\
\hline Satzende & 423 & $24,48 \%$ \\
\hline $\begin{array}{l}\text { Für eine eindeutige } \\
\text { Bestimmung zu kurze Sätze }\end{array}$ & 57 & $3,29 \%$ \\
\hline
\end{tabular}

Tab. 3 | Positionsbestimmung für astheure im Korpus FRAN

Am Beginn oder am Ende eines Satzes werden für gewöhnlich wichtige Informationen platziert. Das bedeutet, dass die Sprecher und Schreiber der mit astheure geleisteten zeitlichen Situierung in ihren Sätzen eine gewisse Wichtigkeit zuschreiben, weshalb sie das Adverb gerne am Anfang oder am Ende ihrer Sätze platzieren. Dass astheure darüber hinaus häufig bei abrupten Themenwechseln verwendet wird, kann ein weiterer Grund dafür sein, dass es häufig am Satzanfang verwendet wird.

Für den anschließenden Vergleich mit maintenant ist zusammenfassend festzustellen, dass das Temporaladverb astheure besonders im oral familier und oral très familier anzutreffen ist. Es wird fast immer zusammen mit dem présent gebraucht und entspricht bisweilen désormais, dorénavant und de nos jours als partiellen Synonymen (cf. zur Terminologie Wunderli 1989, 135-139). Astheure kann des Weiteren zum Vergleich zwischen To und einem anderen Zeitpunkt dienen. Darüber hinaus wird es auch als Diskursmarker verwendet und häufig am Satzanfang oder am Satzende als einer hervorgehobenen Position platziert.

\subsection{Korpusuntersuchung zur Verwendung von maintenant}

Auch für maintenant sei für einen ersten Eindruck eine statistische Übersicht zur Häufigkeit seines Vorkommens in den Korpora gegeben:

\begin{tabular}{|l|l|l|}
\hline Korpus & Anzahl Treffer & Prozentualer Anteil am Korpus \\
\hline FRAN & 1523 & $0,127 \%$ \\
\hline Varitext & 16986 & $0,032 \%$ \\
\hline CFPQ & 98 & $0,014 \%$ \\
\hline
\end{tabular}

Tab. 4 | Statistische Übersicht zum Vorkommen von maintenant in den verwendeten Korpora

Die Tabelle (Tab. 4) zeigt die Anzahl an Belegen für maintenant in den drei verwendeten Korpora. Varitext weist für maintenant mit 16986 Treffern die mit Abstand größte Trefferanzahl auf, allerdings ist der prozentuale Anteil von maintenant am gesamten Korpus dennoch kleiner als der Anteil von maintenant am Korpus FRAN. Den geringsten prozentualen Anteil weist das CFPQ auf. 
Zunächst soll auch für maintenant festgestellt werden, in welchen Varietäten dieses Temporaladverb verwendet wird. Das Adverb weist in den ganz oder zu einem gewichtigen Teil auf mündlich-nähesprachlicher Kommunikation basierenden Korpora CFPQ und FRAN eine ähnlich hohe Trefferquote auf wie astheure, ${ }^{16} \mathrm{dem}$ nach wird auch maintenant sehr häufig in der gesprochenen Sprache verwendet. Dabei zeigt sich im Gegensatz zu astheure eine gewisse Zunahme vom 20. zum 21. Jahrhundert. Die Datierung der Treffer lässt sich mithilfe des Korpus FRAN ermitteln, das eine Filterung der Ergebnisse im Hinblick auf deren Datierung erlaubt:

\begin{tabular}{|l|l|l|}
\hline Zeitraum & Anzahl Treffer für astheure & Anzahl Treffer für maintenant \\
\hline 18.-20. Jahrhundert & 1132 & 79 \\
\hline 21. Jahrhundert & 596 & 1444 \\
\hline
\end{tabular}

Tab. 5 | Datierung der Belege für astheure und maintenant im Korpus FRAN

Ähnlich wie bei astheure können die Briefe aus dem 18. und 19. Jahrhundert, die Treffer für maintenant enthalten, aufgrund der geringen Anzahl an Belegen vernachlässigt werden. Das Pressekorpus Varitext mit seinen 16986 Belegen für maintenant zeigt, dass dieses außer in mündlich-nähesprachlicher auch in schriftlich-distanzsprachlicher Kommunikation häufig vorkommt. Der Einteilung von Langlois $(1999,44)$ folgend könnte man den Gebrauch von maintenant sowohl in die Bereiche écrit soutenu und écrit standard als auch in die Bereiche oral standard und oral familier einordnen. Im Gegensatz zu astheure scheint maintenant demnach keinerlei diasystematischen Restriktionen zu unterliegen.

Auch für maintenant wurden aus den Korpora hundert zufällig ausgewählte Belege hinsichtlich der in den Sätzen verwendeten Verben und des verwendeten Tempus analysiert. Dabei fällt auf, dass die Präsentative c'est und il y a mit maintenant deutlich seltener auftreten. So tritt $c^{\prime}$ est in den hundert ausgewählten Belegen neunmal, il y a hingegen lediglich fünfmal gemeinsam mit maintenant auf. Ferner lassen sich keine Verben feststellen, die besonders häufig mit maintenant erscheinen (auch wenn hier ebenfalls noch eine umfassendere Untersuchung notwendig wäre).

Außerdem erscheint maintenant außer in lediglich elf Fällen ${ }^{17}$ in Sätzen mit finitem Verb im présent. Dies zeigt, dass sich maintenant als deiktisches Element auf den Zeitpunkt des Sprechens bzw. Schreibens bezieht und damit kein Zeitpunkt in der Vergangenheit bestimmt werden kann. Doch in einigen Situationen kann maintenant eine über $T_{0}$ hinausgehende Zukunft einschließen:

\footnotetext{
16 Im Korpus FRAN stammt eine große Anzahl der Belege für astheure aus Louisiana, Massachusetts, Ontario und Nouveau-Brunswick, während deutlich weniger Belege aus Québec stammen. Allerdings sind zurzeit keine genauen Informationen über die quantitative Zusammensetzung dieses Korpus verfügbar (unter https://www.usherbrooke.ca/crifuq/recherche/corpus/corpus-heberges/corpus-fran/ (Stand: 30.01.2021)). Dass astheure allerdings auch in Québec üblich ist und auch dort eine ähnlich hohe Trefferquote aufweist wie maintenant, zeigen die Abfragen des CFPQ. Cf. zum Vorkommen von astheure in Québec auf Grundlage eines Korpus auch Pellicer (1995, 172-173).

17 Diese Zahl ist im Vergleich zu astheure etwas höher, da häufig Formulierungen wie jusqu'à maintenant verwendet werden, die mit dem imparfait einhergehen.
} 
MA1:

a) Maintenant, j'enseigne à Gardner. (FRAN)

b) Désormais, j'enseigne à Gardner.

Die Ersetzbarkeit von maintenant durch désormais in MA1 zeigt, dass maintenant die Zukunft einschließen kann. Dass dies jedoch nicht in allen Fällen funktioniert, zeigt MA2, bei dem sich maintenant nicht durch désormais ersetzen lässt, ohne dass der Satz dadurch ungrammatisch würde:

MA2:

a) Et vous avez encore beaucoup de frères et sœurs maintenant? (FRAN)

b) * Et vous avez encore beaucoup de frères et sours désormais?

Désormais und maintenant sind hier nicht austauschbar, da désormais im Gegensatz zu maintenant nicht mit dem Ausdruck avoir des frères et sœurs verwendet werden kann. Dies liegt darin begründet, dass der Ausdruck im Gegensatz zum semantisch abweichenden être des frères et sœurs nicht mit dem durch désormais ausgedrückten Zeitraum $T_{0}+\infty$ kombiniert werden kann.

Des Weiteren besitzt maintenant wie astheure die Funktion, $T_{0}$ der Vergangenheit gegenüberzustellen:

MA3:

Il est maintenant offert au détail, en librairie, contrairement à son prédécesseur qui n'était disponible que par courtage. (Varitext)

In diesem Fall wird im ersten Teil des Satzes To dargestellt, wohingegen im zweiten Teil durch das imparfait auf einen Zeitraum in der Vergangenheit verwiesen wird.

Für die Erörterung der Position von maintenant im Satz soll nochmals auf das Korpus FRAN zurückgegriffen werden, da dieses Korpus eine einfache Ermittlung der Stellung im Satz zulässt und außerdem eine ausreichend große Anzahl an Treffern für maintenant liefert, sodass mit diesem Korpus diesbezüglich eine relativ verlässliche Aussage getroffen werden kann. Dabei fällt auf, dass maintenant in gut $20 \%$ aller Fälle am Anfang eines Satzes steht, in gut einem Viertel am Satzende und zu ungefähr $50 \%$ in der Satzmitte.

\begin{tabular}{|l|l|l|}
\hline Position & Anzahl Treffer & Prozentualer Anteil \\
\hline Satzbeginn & 296 & $19,44 \%$ \\
\hline Satzmitte & 800 & $52,53 \%$ \\
\hline Satzende & 361 & $23,70 \%$ \\
\hline $\begin{array}{l}\text { Für eine eindeutige } \\
\text { Bestimmung zu kurze Sätze }\end{array}$ & 66 & $4,33 \%$ \\
\hline
\end{tabular}

Tab. 6 | Positionsbestimmung für maintenant im Korpus FRAN

Dies zeigt, dass maintenant zumindest im größtenteils auf mündlich-nähesprachlicher Kommunikation basierenden Korpus FRAN ebenfalls häufig an den Anfang oder das Ende eines Satzes gestellt wird, im Vergleich zu astheure allerdings etwas häufiger in die Satzmitte. Dies lässt darauf schließen, dass die Sprecher maintenant eine etwas weniger wichtige Bedeutung beimessen als astheure. Das könnte darüber hinaus auch darauf hindeuten, dass maintenant im Gegensatz zu astheure 
seltener verwendet wird, um plötzliche Themenwechsel zu vollziehen, weshalb es seltener am Beginn eines Satzes platziert wird. Eine solche Funktion konnte für maintenant auf Grundlage der Korpora nicht festgestellt werden. Es ist zu vermuten, dass solche abrupten Themenwechsel in mündlich-nähesprachlicher Kommunikation häufiger vorkommen als in schriftlich-distanzsprachlicher Kommunikation. Aufgrund seines variationellen Status wird astheure daher häufiger als maintenant verwendet, um abrupte Themenwechsel zu vollziehen. Diese Annahme verstärkt sich bei Betrachtung der Positionierung von maintenant im schriftsprachlichen Korpus Varitext. Hier wird maintenant in lediglich 8,85 \% aller Fälle (1504 von 16986 Treffern) am Satzanfang platziert, während es im vor allem auf mündlich-nähesprachlicher Kommunikation basierenden Korpus FRAN an dieser Stelle in 19,44 \% der Fälle steht.

Gleichwohl kann maintenant wie astheure als Hauptsatzkonjunktion mit adversativer Bedeutung am Satzanfang verwendet werden:

MA4:

L'an passé, quand on avait eu un pic important d'incidences, on avait demandé au laboratoire de santé publique d'étudier nos souches et il nous avait confirmé que la souche A était entrée dans la région, dont à Saint-François d'Assise. Maintenant, cela reste une hypothèse et notre enquête épidémiologique va nous permettre de tirer tout ça au clair. (Varitext)

In MA4 besitzt maintenant keine temporaldeiktische Funktion. Vielmehr dient es hier als Konjunktion, die die beiden Sätze logisch miteinander verbindet und gar eine adversative Bedeutung im Sinne von mais trägt.

\subsection{Vergleich}

Die Gegenüberstellung der Verwendung von astheure und maintenant erbringt neben einer Reihe von Gemeinsamkeiten einige wichtige Unterschiede. Beide Adverbien treten fast ausschließlich mit Verben im présent auf, da sie eine deiktische Funktion des Verweises auf den Moment ausüben, in dem der Satz gesagt oder geschrieben wird $\left(T_{0}\right)$. Dennoch weisen beide nicht in allen Fällen eine zeitliche Beschränkung auf To auf, können bisweilen auch die Zukunft einschließen und so in bestimmten Fällen als Synonyme zu désormais und dorénavant auftreten. Außerdem kann mit ihrer Verwendung ein Zeitpunkt der Vergangenheit To gegenübergestellt werden. Zwar lassen sich für beide Adverbien keine bestimmten Verben ausmachen, die vorzugsweise mit ihnen verwendet werden, dennoch lässt sich bei den Präsentativen c'est und il y a ein wichtiger Unterschied feststellen: Im Gegensatz zu maintenant treten diese Präsentative überdurchschnittlich oft gemeinsam mit astheure auf. Dies stützt die dargelegte diasystematische Situierung von astheure in der mündlich-nähesprachlichen Kommunikation, da derartige Konstruktionen für die Nähesprache kennzeichnend sind.

So besteht der wohl größte Unterschied zwischen astheure und maintenant in den Varietäten, in welchen sie vorkommen. Während maintenant sowohl nähe- als auch distanzsprachlich verwendet wird, ist astheure auf den mündlich-nähesprachlichen Gebrauch beschränkt und wird teilweise sogar als oral très familier 
angesehen. Auch bezüglich der Position der Adverbien im Satz gibt es kleine Unterschiede. Dass astheure häufiger als maintenant am Anfang oder am Ende eines Satzes platziert wird, lässt darauf schließen, dass die Sprecher astheure im Vergleich zu maintenant eine wichtigere Rolle zuschreiben und astheure häufiger für abrupte Themenwechsel nutzen, was wiederum auf den variationellen Status von astheure zurückzuführen ist, da solche plötzlichen Themenwechsel in mündlich-nähesprachlicher Kommunikation häufiger vorkommen. Dennoch kann maintenant wie astheure als Satzkonnektor mit adversativer Bedeutung am Satzanfang platziert werden, wenngleich seine diskursmarkierende Funktion im Gegensatz zu astheure weniger stark konventionalisiert zu sein scheint.

Folgt man den vor allem ab 1976 aufgezeichneten Privatgesprächen im Korpus FRAN, so wird ersichtlich, dass maintenant im 20. Jahrhundert im Französischen Nordamerikas seltener verwendet wurde als astheure, sich diese Situation im 21. Jahrhundert jedoch langsam umkehrt (s. dazu Tab. 5). Dies belegt, dass der Gebrauch von astheure abnimmt, auch wenn es weiterhin tief im französischen Sprachgebrauch Nordamerikas verankert ist.

\section{Fazit}

Insgesamt erweisen sich astheure und maintenant als weitgehend synonym, so zeigen sich bezüglich des mit astheure und maintenant bevorzugt verwendeten Tempus, nämlich des présent, und der Stellung im Satz kaum Unterschiede zwischen den beiden Temporaladverbien, die darüber hinaus beide als Hauptsatzkonjunktionen mit adversativer Bedeutung verwendet werden können. In einer Hinsicht differieren sie jedoch stark voneinander, nämlich bezüglich ihres variationellen Status: Während sich maintenant in allen Varietäten belegen lässt, erweist sich astheure im Französischen Nordamerikas als ein Element der Nähesprache.

Daher bedarf es in einigen Wörterbüchern eines Überdenkens der Einträge zu astheure, denn viele Wörterbücher bewerten astheure lediglich als vieilli, populaire oder régional. In nur wenigen findet sich der Hinweis familier, was sich ändern müsste. Zwar mag es durchaus zutreffend sein, dass die Verwendung von astheure in den letzten Jahrzehnten rückläufig war, dennoch muss bedacht werden, dass es sich dabei nicht um ein veraltetes Wort handelt, sondern um ein regional vorkommendes Synonym von maintenant, das noch heute in Teilen Frankreichs, Belgiens und im gesamten französischsprachigen Teil Nordamerikas verwendet wird. Daher sollte astheure heutzutage weniger als veraltet, sondern eher als umgangs- und regionalsprachlich eingeordnet werden. ${ }^{18}$

Mit dem vorliegenden Aufsatz konnten einige wichtige Fragen zu astheure geklärt werden. In weiteren Arbeiten wäre zu prüfen, wie astheure in anderen Regionen, beispielsweise in einigen Regionen Frankreichs oder Belgiens, verwendet wird und ob sich diesbezüglich Unterschiede zu Nordamerika zeigen. Darüber hinaus würde sich die Auswertung weiterer Korpora lohnen, zum Beispiel solcher mit literarischen

${ }^{18}$ So sieht es im Übrigen auch Avanzi (2019). 
Texten. Da der Gebrauch von astheure rückläufig zu sein scheint, könnte zudem aus soziolinguistischer Perspektive untersucht werden, ob das Adverb von bestimmten Altersgruppen häufiger verwendet wird als von anderen.

\section{Bibliografie}

\section{Quellen}

CFPQ (Corpus du français parlé au Québec), <https://applis.flsh.usherbrooke.ca/cfpq/> (Stand: 29.09.2019).

FRAN (Français d'Amérique du Nord), <http://continent.uottawa.ca/fr/corpus/corpus/corpus-interrogeablefran/> (Stand: 29.09.2019).

TLFQ (Trésor de la langue française au Québec), <http://www.tlfq.ulaval.ca/ilq/> (Stand: 29.09.2019).

Varitext, <http://syrah.uni-koeln.de/varitext/> (Stand: 29.09.2019).

AS1: Varitext: Corpus Presse_Can; Sous-échantillon DEV_Can01; 06.04.2001.

AS2: Varitext: Corpus Presse_Can; Sous-échantillon SOL_Can01; 17.04.2001.

AS3: Varitext: Corpus Presse_Can; Sous-échantillon SOL_CAN01; 15.02.2001.

AS4: Varitext: Corpus Presse_Can; Sous-échantillon DEV_CAN08; 18.12.2008.

AS5 a): FRAN: Conversation avec Suzanne (23 ans) et Adèle (25 ans), Gaston (26 ans) et Jean (27 ans) à Moncton; 1976.

AS6 a): FRAN: Entrevue patrimoniale avec Claude, 56 ans, à Gardner, Massachusetts; 2003.

AS7 a): FRAN: Conversation au Boy's Club avec Chantal (16 ans), Jeanne (16 ans), Lisette (15 ans), Rémi (18 ans), Roger (18 ans) et Roxanne, à Moncton; 1976.

AS8: FRAN: Entrevue patrimoniale variationniste avec Chantal, 61 ans, en Louisiane, Paroise Lafourche; 1997.

AS9: FRAN: Entrevue patrimoniale variationniste, avec Clarisse, 44 ans, Louisiane, Paroise Lafourche; 1997.

AS10: FRAN: Entretien patrimonial avec René (62 ans) Thérèse (61 ans) et Réal (26 ans), à Moncton; 1976.

AS11: FRAN: Entrevue variationniste avec Régine, 75 ans, à Montréal, Hochelaga-Maisonneuve; 2012.

AS12: FRAN: Entrevue patrimoniale variationniste avec Philippe, 27 ans, en Louisiane, Paroise Lafourche, 1997.

AS13: FRAN: Entrevue patrimoniale avec David, 64 ans, en Louisiane, Paroise Lafourche, 1997.

MA1 a): FRAN: Entrevue patrimoniale avec Michel, 63 ans et Jocelyne, 60 ans, à Gardner en Nouvelle-Angleterre; 2003.

MA2 a): FRAN: Entrevue patrimoniale avec Michel, 63 ans et Jocelyne, 60 ans, à Gardner en Nouvelle-Angleterre; 2003.

MA3: Varitext: Corpus Presse_Can; Sous-échantillon DEV_Can01; 17.11.2001.

MA4: Varitext: Corpus Presse_Can; Sous-échantillon DEV_Can07; 14.06.2007. 


\section{Wörterbücher}

DAF1 = Académie française (ed.). 1694. Le Dictionnaire de l'Académie françoise, dedié au Roy, Bd. 1. Paris: Coignard.

DAF3 = Académie française (ed.). ${ }^{3} 1740$. Le Dictionnaire de l'Académie françoise, Bd. 1. Paris: Coignard.

DC = Dulong, Gaston (ed.). 1989. Dictionnaire des canadianismes. Montréal: Larousse Canada.

DCF = Robinson, Sinclair \& Donald Smith (ed.). 1991. NTC's dictionary of Canadian French. Lincolnwood: NTC.

$\mathrm{DCL}=$ Daigle, Jules O. (ed.). 1984. A dictionary of the Cajun language. Ville Platte: Swallow Publications.

DCLF = Rey, Alain (ed.). 2005. Dictionnaire culturel en langue française, Bd. 2. Paris: Dictionnaires Le Robert.

DECFA = Bollée, Annegret et al. (ed.). 2018. Dictionnaire étymologique des créoles français d'Amérique, Bd. 2. Hamburg: Buske.

DEFFQ = Beauchemin, Normand (ed.). 1982. Dictionnaire d'expressions figurées en français parlé du Québec. Les 700 "québécoiseries» les plus usuelles. Sherbrooke: Université.

DFQ = Poirier, Claude (ed.). 1985. Dictionnaire du français québécois. Description et histoire des régionalismes en usages au Québec. Sainte-Foy: Les Presses de I'Université Laval.

$\mathrm{DH}=$ Fouquet, Emmanuel \& Élisabeth Bonvarlet (ed.). 2000. Dictionnaire Hachette langue française. Paris: Hachette.

DHFQ = Poirier, Claude (ed.). 1998. Dictionnaire historique du français québécois. Sainte-Foy: Les Presses de l'Université Laval.

DLFC = Bélisle, Louis-Alexandre (ed.). 1974. Dictionnaire général de la langue française au Canada. Québec: Bélisle [u. a.].

$\mathrm{DLQ}=$ Bergeron, Léandre (ed.). 1980. Dictionnaire de la langue québécoise. Montréal: VLB Éditeur.

DLQR = Rogers, David (ed.). 1977. Dictionnaire de la langue québécoise rurale. Montréal: VLB Éditeur.

DQA = Boulanger, Jean-Claude (ed.). 1993. Dictionnaire québécois d'aujourd'hui. Langue française, histoire, géographie, culture générale. Saint-Laurent: Dicorobert.

DQF = Meney, Lionel (ed.). 1999. Dictionnaire québécois-français. Pour mieux se comprendre entre francophones. Montréal: Guérin.

DRF = Rézeau, Pierre (ed.). 2001. Dictionnaire des régionalismes de France. Géographie et histoire d'un patrimoine linguistique. Brüssel: De Boeck [u. a.].

DRFT = Brasseur, Patrice (ed.). 2001. Dictionnaire des régionalismes $d u$ français de Terre-Neuve. Tübingen: Niemeyer.

DRIM = Naud, Chantal (ed.). 2011. Dictionnaire des régionalismes des îles de la Madeleine. Montréal: Les Éditions Québec Amérique inc.

GPFC = Société du parler français au Canada (ed.). 1968. Glossaire du parler français au Canada. Québec: Les Presses de l'Université Laval.

FEW = Wartburg, Walther von (ed.). 1952. Französisches etymologisches Wörterbuch, Bd. 4. Basel: R. G. Zbinden \& CO.

NPR $=$ Rey-Debove, Josette \& Alain Rey (ed.). 2002. Le nouveau Petit Robert. Dictionnaire alphabétique et analogique de la langue française. Paris: Dictionnaires Le Robert. 


\section{Literatur}

AvanzI, Mathieu. 2019. „Astheure (à c't’heure). "In Français de nos régions. $<$ https://francaisdenosregions.com/2019/07/11/astheure/> (14.01.2021).

BEIßWENGER, Michael. 2016. „Sprache und Medien. Digitale Kommunikation.“ In Studiport Sprach- und Textverständnis. Hypermediales E-LearningAngebot des Ministeriums für Innovation, Wissenschaft und Forschung (MIWF) des Landes Nordrhein-Westfalen.

$<$ http://www.michael-beisswenger.de/pub/beisswenger_digikomm_preview.pdf> (17. 01.2021).

Bergeron-MaguiRe, Myriam. 2011. La "conscience diasporale " en poésie cadienne.

<https://corpus.ulaval.ca/jspui/bitstream/20.500.11794/22973/1/28724.pdf> (29.09. 2019).

BERTIN, Annie. 2001. "Maintenant. Un cas de grammaticalisation ? "Langue française 130, 42-64.

FRANK-JOB, Barbara. 2010. „Die Entwicklung deiktischer Ausdrücke zu Diskursmarkern im Kontext von Interaktionsanalyse und Sprachwandelforschung." In Wenn Deiktika nicht zeigen. Zeigende und nichtzeigende Funktionen deiktischer Formen in den romanischen Sprachen, ed. Maaß, Christiane \& Angela Schrott, 283-308, Münster: LIT.

GrevISSE, Maurice \& André Goosse (ed.). ${ }^{15} 2011$. Le bon usage. Grammaire française. Brüssel: De Boeck, Duculot.

HeRBST, Thomas \& Michael Klotz. 2003. Lexikografie. Paderborn [u. a.]: Schöningh.

KNAZ, Wissem. 2015. „Le langage utilisé sur les réseaux sociaux. L'émergence d'une nouvelle communauté linguistique." Revue Internationale 1/2, <https://revues.imist.ma/index.php/Revue-Interdisciplinaire/article/view/4053/2986> (17.01.2021).

$\mathrm{KoCH}$, Peter \& Wulf Oesterreicher. ${ }^{2} 2011$. Gesprochene Sprache in der Romania. Französisch, Italienisch, Spanisch. Berlin/New York: De Gruyter.

LANGLOIS, Marie-France. 1999. Une nouvelle grille de marques de registres de langue: perception et usage des rédacteurs et communicateurs québécois. Sherbrooke: Université de Sherbrooke.

LECLERC, Jacques. 2017. „La Nouvelle-France (1534-1760). L'implantation du français au Canada." In L'aménagement linguistique dans le monde. Québec: CEFAN, Université Laval.

$<$ http://www.axl.cefan.ulaval.ca/francophonie/HISTfrQC_s1_Nlle-France.htm> (16.01. 2021).

MARTUCCI, Jean. 1988. „Le français du Québec." In Actes du XVIIIe Congrès International de Linguistique et de Philologie Romanes. Université de Trèves 1986, Bd. 5, ed. Kremer, Dieter, 102-110, Tübingen: Niemeyer.

Matus-MendOZA, Mariadelaluz. 2000. „Ahora' (now) and ,ahorita' (right now) as deictic markers." In National association of African American studies \& national association of Hispanic and Latino studies, 69-81, Houston: Language Issues Monograph.

MAZIERE, Francine. 1995. „Le Dictionnaire de l'Académie française (1694). Initiation d'une pratique normative." Archives et documents de la Société d'histoire et d'épistémologie des sciences du langage 11, 12-17.

MELANÇON, Benoît. 2016. Maintenant. <https://oreilletendue.com/2016/02/24/maintenant/> (29.09.2019).

MerCier, Louis. 2002. La Société du parler français au Canada et la mise en valeur du patrimoine linguistique québécois. Histoire de son enquête et genèse de son glossaire. Québec: Les Presses de l'Université Laval.

MoRvan, Malo. 2019. "L'Académie, greffier du bon usage ? Exploiter la polysémie de la notion d'usage pour construire un ethos d'autorité." In 
Les discours de référence sur la langue française, ed. Dister, Anne \& Sophie Piron, 283-309, Brüssel: Presses de l'Université Saint-Louis.

OVERBECK, Anja. 2012. „,Parlez-vous texto?' Soziale Netzwerke an der Schnittstelle zwischen realem und virtuellem Raum." In Sprache und Öffentlichkeit in realen und virtuellen Räumen. Akten der Sektion auf dem 7. Kongress des Frankoromanistenverbands (Essen, 29.9.-2.10.2010), ed. Gerstenberg, Annette, Claudia Polzin-Haumann \& Dietmar Osthus, 217247, Bonn: Romanistischer Verlag.

PELLICER, Dora. 1995. „,Qu'est ce qui t'ammène icitte asteure ?’ Archaïsmes, anglicismes et innovations du français québécois." Estudios de Lingüística Aplicada 21/22, 150-182.

Peronnet, Louise. 1989. Le parler acadien du Sud-Est du Nouveau-Brunswick. Éléments grammaticaux et lexicaux. New York: Lang.

PoIRIER, Claude. 1995. „Les variantes topolectales du lexique français. Propositions de classement à partir d'exemples québécois." In Le régionalisme lexical, ed. Francard, Michel \& Danièle Latin, 13-56, Louvainla-Neuve: Duculot.

PöLL, Bernhard. 22017. Französisch außerhalb Frankreichs. Geschichte, Status und Profil regionaler und nationaler Varietäten. Berlin/Boston: De Gruyter.

REMYSEN, Wim. 2003. "Le français au Québec. Au-delà des mythes." Romaneske 28, 28-41.

SCHAFROTH, Elmar. 2014. Französische Lexikografie. Einführung und Überblick. Berlin [u. a.]: De Gruyter.

SEGUIN, Aude. 2011. "Désormais, dorénavant et astheure. Variation aspectuo-temporelle." Communication, lettres et sciences du langage 5, 137-147.

<https://clsl.re-cherche.usherbrooke.ca/vol5no1/SEGUIN_vol5_no1_2011.pdf> (29.09. 2019).

Simoni-Aurembou, Marie-Rose. 2004. „Les apports du sud-ouest de la région parisienne aux régionalismes de la France." In Français du Canada Français de France. Actes du sixième Colloque international d'Orford, Québec, du 26 au 29 septembre 2000, ed. Mercier, Louis, 59-70, Tübingen: Niemeyer.

StÄBLER, Cynthia K. 1995a. La vie dans le temps et asteur. Ein Korpus von Gesprächen mit Cadiens in Louisiana. Tübingen: Narr.

STÄBLER, Cynthia K. 1995b. Entwicklung mündlicher romanischer Syntax. Das français cadien in Louisiana. Tübingen: Narr.

THIBAULT, André. 2009. „Français d’Amérique et créoles / français des Antilles. Nouveaux témoignages." Revue de linguistique romane 73, 77-137.

THIBAULT, André. 2015. Du français aux créoles. Phonétique, lexicologie et dialectologie antillaises. Paris: Classiques Garnier.

THIBAULT, André. 2016. „Diastratismes et réallocation des variantes. Français d'Amérique et de Nouvelle-Calédonie." Langages 203 (3), 71-86.

VoGH, Kendall. 2018. Ressources linguistiques et visée référentielle chez les individus bilingues français-anglais. L'alternance codique comme stratégie d'expression sur le plan lexical.

$<$ https://corpus.ulaval.ca/jspui/bitstream/20.500.11794/29802/1/34039.pdf> (29.09. 2019).

WUNDERLI, Peter. 1989. Französische Lexikologie. Einführung in die Theorie und Geschichte des französischen Wortschatzes. Tübingen: Niemeyer. 


\title{
Zusammenfassung
}

Der Aufsatz untersucht die Verwendung der beiden französischen Temporaladverbien astheure und maintenant im Französischen Nordamerikas. Aufbauend auf einer Analyse von Wörterbucheinträgen zum Adverb astheure wird zunächst dessen Verbreitung in Frankreich und Nordamerika aufgezeigt, wo astheure weitaus gebräuchlicher ist als in Frankreich selbst. Im zweiten Teil des Aufsatzes wird die Verwendung von astheure im Vergleich zu maintenant anhand einer Korpusuntersuchung analysiert, die auf den drei Korpora FRAN, Varitext und CFPQ basiert. Dabei wird ersichtlich, dass sich die beiden Adverbien zwar weitgehend als synonym erweisen, hinsichtlich ihres variationellen Status jedoch stark voneinander unterscheiden: Während sich maintenant in allen Varietäten belegen lässt, erweist sich astheure im Französischen Nordamerikas als ein Element der Nähesprache.

\begin{abstract}
The article examines the use of the French temporal adverbs astheure and maintenant in the French-speaking part of North America. Based on an analysis of dictionary entries of the adverb astheure, the article initially describes its distribution in France and North America, where astheure is by far more often used than in France. The second part of the article compares the usage of astheure and maintenant in North America on the basis of a corpus study. This study, which is based on the three corpora FRAN, Varitext and CFPQ, clearly shows that the two adverbs can largely be seen as synonyms, but that there is an important difference with regard to their variational status: Whereas maintenant is used in all varieties, astheure proves to be an element of the language of proximity in North American French.
\end{abstract}

